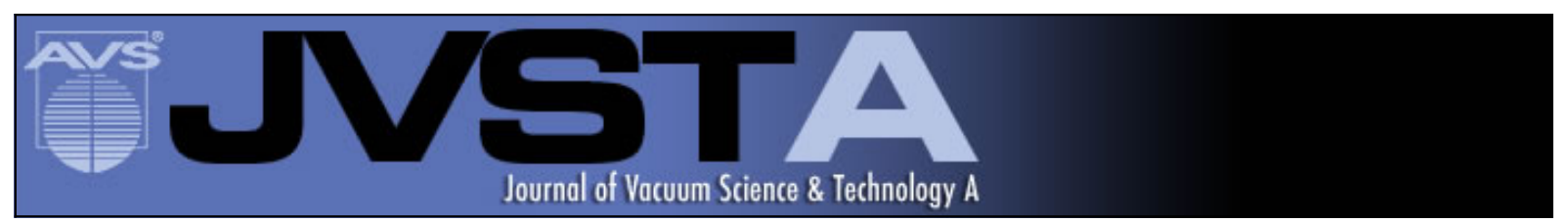

\title{
Fluid simulation of the bias effect in inductive/capacitive discharges
}

Yu-Ru Zhang, Fei Gao, Xue-Chun Li, Annemie Bogaerts, and You-Nian Wang

Citation: Journal of Vacuum Science \& Technology A 33, 061303 (2015); doi: 10.1116/1.4928033

View online: http://dx.doi.org/10.1116/1.4928033

View Table of Contents: http://scitation.aip.org/content/avs/journal/jvsta/33/6?ver=pdfcov

Published by the AVS: Science \& Technology of Materials, Interfaces, and Processing

\section{Articles you may be interested in}

Particle-in-cell simulations of hollow cathode enhanced capacitively coupled radio frequency discharges

Phys. Plasmas 19, 023508 (2012); 10.1063/1.3685709

Phase-shift effects on growth and transport of dust particles in VHF capacitively coupled silane discharges: Two dimensional fluid simulation

Phys. Plasmas 18, 083508 (2011); 10.1063/1.3626544

Verification of collisionless sheath model of capacitive rf discharges by particle-in-cell simulations

J. Appl. Phys. 110, 033307 (2011); 10.1063/1.3620983

Comparison of atmospheric-pressure helium and argon plasmas generated by capacitively coupled radiofrequency discharge

Phys. Plasmas 13, 093503 (2006); 10.1063/1.2355428

Modeling of ionization of argon in an analytical capacitively coupled radio-frequency glow discharge J. Appl. Phys. 86, 2990 (1999); 10.1063/1.371159

\section{Instruments for Advanced Science} w www.HidenAnalytical.com E info@hiden.co.uk CLICK TO VIEW our product catalogue

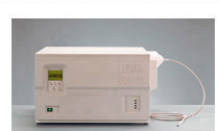

Gas Analysis

dynamic measurement of reaction gas streans catalysis and thermal analysis molecular beam studies

dissolved species probes

, fermentation environmental and ecological studes

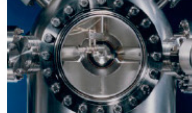

Surface Science

UHVTPD

SIMS emental imaging - surface mapping

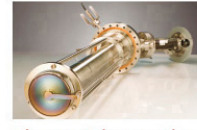

Plasma Diagnostics plasma source characterization etch and deposition process reaction , analysis of neutral and radical species

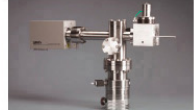

Vacuum Analysis partial pressure measurement and contro of process gases reactive sputter process contro vacuum diagnostics Toum coating prossmonitorin 


\title{
Fluid simulation of the bias effect in inductive/capacitive discharges
}

\author{
Yu-Ru Zhang \\ Key Laboratory of Materials Modification by Laser, Ion, and Electron Beams (Ministry of Education), \\ School of Physics and Optoelectronic Technology, Dalian University of Technology, Dalian 116024, \\ China and Research Group PLASMANT, Department of Chemistry, University of Antwerp, \\ Universiteitsplein 1, Wilrijk, BE-2610 Antwerp, Belgium
}

Fei Gao and Xue-Chun Li

Key Laboratory of Materials Modification by Laser, Ion, and Electron Beams (Ministry of Education), School of Physics and Optoelectronic Technology, Dalian University of Technology, Dalian 116024, China

\author{
Annemie Bogaerts \\ Research Group PLASMANT, Department of Chemistry, University of Antwerp, Universiteitsplein 1, Wilrijk, \\ BE-2610 Antwerp, Belgium \\ You-Nian Wang ${ }^{\text {a) }}$ \\ Key Laboratory of Materials Modification by Laser, Ion, and Electron Beams (Ministry of Education), \\ School of Physics and Optoelectronic Technology, Dalian University of Technology, Dalian 116024, China
}

(Received 16 April 2015; accepted 24 July 2015; published 7 August 2015)

\begin{abstract}
Computer simulations are performed for an argon inductively coupled plasma (ICP) with a capacitive radio-frequency bias power, to investigate the bias effect on the discharge mode transition and on the plasma characteristics at various ICP currents, bias voltages, and bias frequencies. When the bias frequency is fixed at $13.56 \mathrm{MHz}$ and the ICP current is low, e.g., $6 \mathrm{~A}$, the spatiotemporal averaged plasma density increases monotonically with bias voltage, and the bias effect is already prominent at a bias voltage of $90 \mathrm{~V}$. The maximum of the ionization rate moves toward the bottom electrode, which indicates clearly the discharge mode transition in inductive/capacitive discharges. At higher ICP currents, i.e., 11 and $13 \mathrm{~A}$, the plasma density decreases first and then increases with bias voltage, due to the competing mechanisms between the ion acceleration power dissipation and the capacitive power deposition. At $11 \mathrm{~A}$, the bias effect is still important, but it is noticeable only at higher bias voltages. At $13 \mathrm{~A}$, the ionization rate is characterized by a maximum at the reactor center near the dielectric window at all selected bias voltages, which indicates that the ICP power, instead of the bias power, plays a dominant role under this condition, and no mode transition is observed. Indeed, the ratio of the bias power to the total power is lower than 0.4 over a wide range of bias voltages, i.e., $0-300 \mathrm{~V}$. Besides the effect of ICP current, also the effect of various bias frequencies is investigated. It is found that the modulation of the bias power to the spatiotemporal distributions of the ionization rate at $2 \mathrm{MHz}$ is strikingly different from the behavior observed at higher bias frequencies. Furthermore, the minimum of the plasma density appears at different bias voltages, i.e., $120 \mathrm{~V}$ at $2 \mathrm{MHz}$ and $90 \mathrm{~V}$ at $27.12 \mathrm{MHz}$. (ㅇ 2015 American Vacuum Society. [http://dx.doi.org/10.1116/1.4928033]
\end{abstract}

\section{INTRODUCTION}

Inductively coupled plasma (ICP) sources are being widely used in the semiconductor manufacturing industry, as they are characterized by several advantages, such as high plasma density at low gas pressure, relative simplicity, no requirement for external magnetic fields, and so on. ${ }^{1,2}$ For etching and deposition processes, it is important to control the plasma density and ion bombardment energy independently, which is difficult to be realized in purely inductive discharges. Therefore, a radio-frequency (RF) bias power is often employed and capacitively coupled to the substrate..$^{3-7}$ In this discharge system, the plasma is generated by the ICP power, which controls the plasma density and ion flux, and the capacitive bias power is used to control the energy of the ions at the substrate. The bias power, which determines the ion energy distribution (IED) at the wafer, has a significant

$\overline{{ }^{a)} \text { Electronic mail: ynwang@dlut.edu.cn }}$ influence on the plasma properties and is, for instance, responsible for the etch rate. Hence, the bias power effect should be systematically investigated, in order to control the discharge process and to optimize the plasma for microelectronics applications.

In recent years, significant progress has been achieved in experimental studies of the bias effect in inductive discharges with a biased electrode. ${ }^{8-13}$ Rauf et al. ${ }^{8}$ measured the electron density and total negative ion density in $\mathrm{Ar} / \mathrm{SF}_{6}$ plasmas, by using microwave interferometry and laser photodetachment, respectively. Sobolewski and $\mathrm{Kim}^{9}$ performed measurements with a cutoff probe and a Langmuir probe, and reported a bias-induced decrease in the electron density at high ICP powers in $\mathrm{Ar}, \mathrm{CF}_{4}$, and $\mathrm{Ar} / \mathrm{CF}_{4}$ plasmas. However, when the ICP power is low, a strikingly different evolution was observed, and the RF bias produced an increase in the electron density. Lee et al. ${ }^{10}$ revealed that the plasma density increased considerably with bias power when the discharge was in capacitive mode (E mode), whereas a 
decreasing trend was observed in the inductive mode $(\mathrm{H}$ mode), which was in good agreement with the experimental results presented in Ref. 9. Last year, Schulze et al. ${ }^{11}$ verified experimentally, by phase resolved optical emission spectroscopy as well as current and voltage measurements, that three coupling mechanisms (i.e., sheath heating due to the bias power, the substrate sheath voltage affected by the ICP power, and the amplitude as well as the frequency of plasma series resonance oscillations of the RF current affected by the ICP power) were potentially limiting the separate control of the ion energy and flux in a neon ICP discharge with RF bias. Subsequently, Lee et al. ${ }^{12}$ performed measurements in an ICP reactor with bias frequency of 12.5 $\mathrm{MHz}$ and came to the conclusion that the plasma density near the radial edge had a pronounced increase, and therefore, the plasma uniformity was improved significantly when a bias power was applied. Furthermore, Lee and Chung ${ }^{13}$ discovered that the high energy electrons were generated by the capacitively coupled collisionless heating of the electron bounce resonance, when a high RF bias power was coupled to the substrate.

In addition, some theoretical research has also been put forward to investigate the bias effect in a combined plasma reactor with both ICP power and RF bias. ${ }^{8,14-19}$ Rauf et al. ${ }^{8}$ employed the hybrid plasma equipment model (HPEM) with a detailed plasma chemistry description and showed that the RF bias power had very little effect on the ion and electron densities in an $\mathrm{Ar} / \mathrm{SF}_{6}$ discharge. Hoekstra and Kushner ${ }^{14}$ focused on the IED by using the HPEM coupled with the plasma chemistry Monte Carlo simulation module. They discovered that the shape of the IED depends both on the amplitude of the RF bias (which determines the average energy of the IED) and on the ICP power (which determines the width of the IED). Takekida and Nanbu ${ }^{15}$ utilized a particle-in cell Monte Carlo method, showing that the uniformity of the ion flux could be improved by decreasing the bias frequency. Tinck et al. ${ }^{16}$ illustrated with the HPEM that the energy and fluxes of the ions hitting the substrate became higher at increasing bias, and this gave rise to a higher etch rate. Kwon et al. ${ }^{17}$ presented a self-consistent global model combined with a sheath module to examine the bias power effect on the plasma properties. Finally, Zhang and Kushner ${ }^{18}$ and Rauf $^{19}$ investigated the influence of the bias power on the etch rate in $\mathrm{C}_{2} \mathrm{~F}_{6}$ and $\mathrm{CF}_{4} / \mathrm{O}_{2}$ plasmas, respectively.

Indeed, the bias power can penetrate into the plasma bulk, contribute to the electron heating, and therefore change the plasma characteristics. From the literature overview mentioned above, it is clear that quite a lot of research has been carried out already on the bias effect in ICP discharges, but there still remain some questions, especially regarding the effect on the discharge mode transition. In the previous investigations, the emphasis was mainly put on the IED (Refs. 14-16) and etch rate ${ }^{18,19}$ in the ICP reactor with a biased substrate. Although the plasma density has been reported as a function of bias power in Refs. 8 and 17, the qualitative trend was not in good agreement with the experimental results observed in Ref. 13, and the mechanisms behind the bias effect are still not clear. When a capacitive rf bias is applied to the substrate, an rf voltage drop is produced at the boundary of the plasma, and the plasma potential will be modulated. Moreover, the electrons can be heated not only by the inductive power, but also by the rf sheath oscillation, and hence, the balance between the power absorption and power dissipation will be changed, and this has a direct influence on the plasma properties. In order to obtain optimal device performance during the etching process, the plasma parameters must be carefully controlled, so it is of great importance to elucidate the bias effect on the plasma characteristics. Therefore, a comprehensive, detailed study of the bias effect on the discharge mode transition and on the plasma properties, especially under different discharge conditions, should be carried out. In this paper, the so-called Multiphysics Analysis for Plasma Sources-ICP (MAPS-ICP) solver ${ }^{25}$ accompanied with the MAPS-sheath module ${ }^{35}$ has been employed, to investigate the bias effect on the discharge mode transition and plasma characteristics in an ICP reactor at various ICP currents, bias voltages, and bias frequencies. Indeed, it is very important to have a good insight in the bias effect, because it has a significant influence on the plasma properties and plays a key role in plasma processing applications.

\section{DESCRIPTION OF THE FLUID MODEL}

A schematic of the ICP reactor with capacitively coupled bias power, studied in this work, is shown in Fig. 1. The reactor is cylindrical, with a radius of $15 \mathrm{~cm}$. The discharge is sustained by a two-turn planar coil, which is separated from the plasma by a dielectric window. The radii of the inner and outer turns of the coil are 6 and $8 \mathrm{~cm}$, respectively. The bottom electrode with a radius of $13 \mathrm{~cm}$ is fixed at a distance of $9 \mathrm{~cm}$ from the quartz window and is biased by a RF power to independently control the ion bombardment energy.

MAPS is a comprehensive modeling platform developed by Prof. Wang and his group for the multiphysics analysis of various plasma sources. It consists of a series of modules,

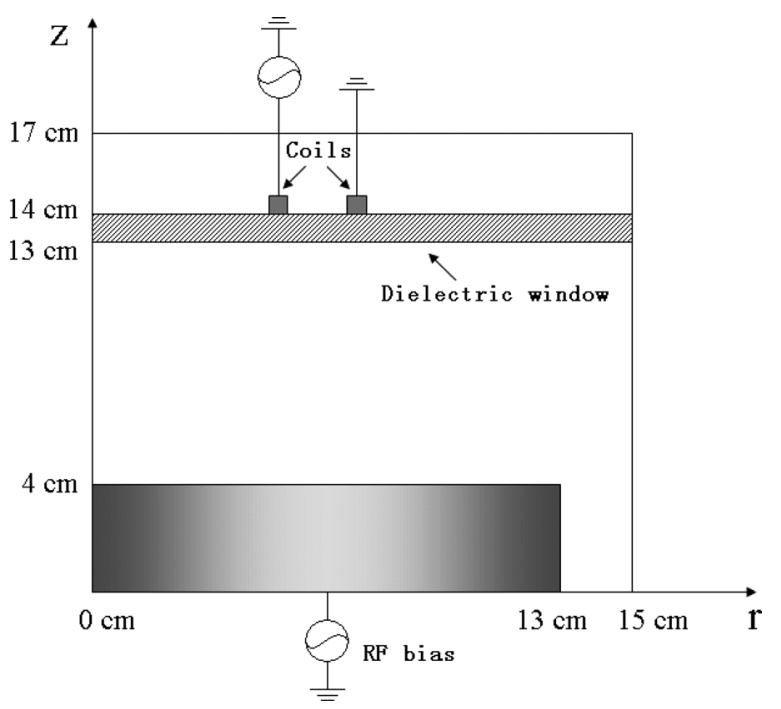

FIG. 1. Schematic picture of the cylindrical inductively coupled plasma reactor configuration. 
which are iterated to reach a converged solution. MAPS includes two solvers: the MAPS-CCP solver and the MAPSICP solver. The MAPS-CCP solver, which is used to investigate the multiphysical coupling phenomena in capacitively coupled discharges, is composed of a two-dimensional fluid model $^{20,21}$ and a particle-in-cell/Monte Carlo method. ${ }^{22,23}$ For the MAPS-ICP solver, only the two-dimensional fluid model is employed, ${ }^{24,25}$ focusing on the discharge properties in inductively coupled plasmas. To complete the MAPS, some other modules are also included, for instance, the input module, the output module, the reaction chemistry module, the electromagnetic module, and the sheath module. The MAPS-sheath module can be used to describe the spatiotemporal characteristics of the RF sheath. Except for the modules mentioned above, other functions of the MAPS are currently under development.

In this paper, the MAPS-ICP solver combined with the MAPS-sheath module is employed to investigate the bias effect on the discharge mode transition and plasma characteristics in a combined discharge with both inductive and bias powers. The modules are described as follows.

\section{A. Input module}

Before we start the simulation, the discharge parameters and the reactor geometry should be defined as input in the model. The discharge parameters consist of three parts: source parameters, gas parameters, and numerical treatment parameters. In the source parameter part, the amplitudes and frequencies of the ICP power and RF bias are defined, as well as the phase shift value between them. Subsequently, the type of gas, gas pressure, and gas mixing ratio are chosen in the gas parameter section. Finally, numerical treatment parameters, which are responsible for the accuracy, stability, and efficiency of the numerical simulation, should also be given as input. These parameters include initial values of the plasma properties (i.e., the plasma density, electron temperature, and so on), the time step, and iteration factors of successive-over-relaxation (SOR), and other algorithms.

In addition, the reactor geometry is also an important input factor in the simulation. It includes the dimensions of the electrode and the discharge region, the relative dielectric constant of the dielectric window, and the position of the coil. Besides, the boundary conditions, which include the axis boundary, source boundary, grounded boundary, dielectric boundary, and outflow boundary, must be specified in order to complete the problem.

\section{B. Reaction chemistry module}

There exist various kinds of plasma species in the discharge, including molecules, radicals, positive and negative ions, excited species, as well as electrons. These species react with each other in a large number of collisions, and therefore, a list of all the important chemical reactions is constructed in the reaction chemistry module, including electron-impact (electron-neutral and electron-ion) collisions and heavy particle (i.e., ion-ion, ion-neutral, and neutral- neutral) reactions. For all these reactions, energy-dependent cross sections (for the electron-impact collisions) and rate coefficients (for the heavy particle reactions) are collected, and are used as input into the source terms, which will be used for solving the continuity equations. Besides, the transport coefficients (i.e., diffusion coefficients for the plasma species, as well as the viscosity and the thermal conductivity for a pure gas or gas mixtures) are also defined in this module.

In this work, the simulations were performed for an $\mathrm{Ar}$ plasma, and the species considered in the model include the electrons, $\mathrm{Ar}^{+}$, and $\mathrm{Ar}^{*}$. All the reactions taken into account in the model are presented in Table I.

\section{Plasma module}

In the plasma module, which forms the core of the MAPS-ICP solver, the plasma is treated as a continuum, which consists of a large number of plasma species, including various molecules, radicals, ions, excited species, as well as electrons. The average quantities of these particles, such as density, mean velocity, and mean energy, can be obtained by solving the moments of the Boltzmann equation, which are formed by multiplying the Boltzmann equation by $\mathbf{v}^{0}, \mathbf{v}^{1}, \mathbf{v}^{2}$, and integrating over velocity.

We solve the equations of continuity and energy for electrons

$$
\begin{aligned}
& \frac{\partial n_{e}}{\partial t}+\nabla \cdot \boldsymbol{\Gamma}_{\mathbf{e}}=S_{e} \\
& \frac{\partial\left(\frac{3}{2} n_{e} k_{B} T_{e}\right)}{\partial t}=-\nabla \cdot \mathbf{q}_{\mathbf{e}}-e \mathbf{E}_{\mathbf{s}} \cdot \boldsymbol{\Gamma}_{\mathbf{e}}+P_{\text {ind }}-W_{e},
\end{aligned}
$$

TABLE I. Chemical reactions included in the model.

\begin{tabular}{lcc}
\hline \hline Process & Reaction & Rate coefficient $\left(\mathrm{cm}^{3} / \mathrm{s}\right)$ \\
\hline Ground state ionization & $\mathrm{e}+\mathrm{Ar} \rightarrow 2 \mathrm{e}+\mathrm{Ar}^{+}$ & $\mathrm{a}$ \\
Ground state excitation & $\mathrm{e}+\mathrm{Ar} \rightarrow \mathrm{e}+\mathrm{Ar}^{*}$ & 36 \\
Stepwise ionization & $\mathrm{e}+\mathrm{Ar}^{*} \rightarrow 2 \mathrm{e}+\mathrm{Ar}^{+}$ & $\mathrm{a}$ \\
Superelastic collision & $\mathrm{e}+\mathrm{Ar}^{*} \rightarrow \mathrm{e}+\mathrm{Ar}$ & $\mathrm{a}$ \\
Quenching to resonant; radiative decay & $\mathrm{e}+\mathrm{Ar}^{*} \rightarrow \mathrm{e}+\mathrm{Ar}$ & $\mathrm{a}$ \\
Metastable pooling & $\mathrm{Ar}^{*}+\mathrm{Ar}^{*} \rightarrow \mathrm{e}+\mathrm{Ar}+\mathrm{Ar}$ & $38^{\mathrm{b}}$ \\
Two-body quenching & $\mathrm{Ar}+\mathrm{Ar}^{*} \rightarrow 2 \mathrm{Ar}$ & $39^{\mathrm{b}}$ \\
\hline \hline
\end{tabular}

${ }^{a}$ The rate coefficient is calculated from the corresponding collision cross section.

${ }^{\mathrm{b}} \mathrm{Ar}{ }^{*}$ represents a composite metastable level consisting of ${ }^{3} \mathrm{P}_{0}$ and ${ }^{3} \mathrm{P}_{2}$. 
where the electron flux $\Gamma_{\mathrm{e}}$ can be presented in the driftdiffusion form

$$
\boldsymbol{\Gamma}_{\mathbf{e}}=-\frac{1}{m_{e} \nu_{\mathrm{en}}} \nabla\left(n_{e} k_{B} T_{e}\right)-\frac{e n_{e}}{m_{e} \nu_{\mathrm{en}}} \mathbf{E}_{\mathbf{s}}
$$

and the flux of energy $\mathbf{q}_{\mathbf{e}}$ is expressed as

$$
\mathbf{q}_{\mathbf{e}}=\frac{5}{2} k_{B} T_{e} \boldsymbol{\Gamma}_{\mathbf{e}}-\frac{5}{2} \frac{n_{e} k_{B} T_{e}}{m_{e} \nu_{\mathrm{en}}} \nabla\left(k_{B} T_{e}\right)
$$

Here, $n_{e}, m_{e}$, and $T_{e}$ are the density, mass, and temperature of the electrons, respectively; $\mathbf{E}_{\mathbf{s}}$ is the static electric field; $\nu_{\mathrm{en}}$ is the collision frequency between electrons and neutral particles; $k_{B}$ is the Boltzmann constant; $S_{e}$ is the source of electrons; $W_{e}=\sum_{j} \varepsilon_{j} k_{j} n_{1} n_{2}$ is the energy exchange during all the collisional processes; and $k_{j}$ is the reaction coefficient. Positive values of $\varepsilon_{j}$ imply energy loss by electrons (e.g., ionization), and negative values imply energy gain (e.g., superelastic collisions). The period averaged inductive heating term $P_{\text {ind }}$ is described as $P_{\text {ind }}=1 / T \int_{0}^{T} J_{\theta} E_{\theta} d t$.

The behavior of the ions is governed by the continuity equation and momentum balance equation. Since the ions are assumed to be at room temperature, no energy balance equation needs to be solved for them

$$
\begin{aligned}
& \frac{\partial n_{i}}{\partial t}+\nabla \cdot\left(n_{i} \mathbf{u}_{\mathbf{i}}\right)=S_{i} \\
& \frac{\partial\left(n_{i} m_{i} \mathbf{u}_{\mathbf{i}}\right)}{\partial t}+\nabla \cdot\left(n_{i} m_{i} \mathbf{u}_{\mathbf{i}} \mathbf{u}_{\mathbf{i}}\right)=-\nabla p_{i}+Z_{i} e n_{i} \mathbf{E}_{\mathbf{s}}+\mathbf{M}_{\mathbf{i}} .
\end{aligned}
$$

Here, $n_{i}, \mathbf{u}_{\mathbf{i}}, S_{i}, m_{i}$, and $Z_{i}$ are the ion density, velocity, source term, mass, and charge, respectively; $p_{i}$ is the ion pressure; and $\mathbf{M}_{\mathbf{i}}$ represents the collisional transfer of momentum from ions to neutral species. ${ }^{26}$

The electrostatic fields due to the space charge within the plasma, which in turn influence the charged particle behavior, can be determined by solving the Poisson's equation

$$
\nabla^{2} \phi=\frac{e}{\varepsilon_{0}}\left(n_{e}-\sum Z_{+} n_{+}+\sum Z_{-} n_{-}\right),
$$

where $\phi$ is the electric potential and $\varepsilon_{0}$ is the vacuum permittivity.

The electron flux at the electrodes and side walls is given by using the kinetically limited Maxwellian flux condition $\Gamma_{\mathbf{e}}= \pm 1 / 4 n_{e} \mathbf{u}_{\mathbf{e}, \mathrm{th}} \exp \left(\phi_{b} / k_{B} T_{e}\right)$, where the \pm signs correspond to the direction of the electron flux, $\phi_{b}$ is the boundary potential, and $\mathbf{u}_{\mathbf{e}, \mathrm{th}}$ is the electron thermal velocity, expressed as $\mathbf{u}_{\mathbf{e}, \mathbf{t h}}=\left(8 k_{B} T_{e} / \pi m_{e}\right)^{1 / 2}$. Electron reflection at the boundaries is accounted for, with a reflection coefficient $\Theta$ of 0.25 . Ignoring the electron thermal conduction, the energy flux to the electrodes and walls is set to $\mathbf{q}_{\mathbf{e}}=5 / 2 k_{B} T_{e} \boldsymbol{\Gamma}_{\mathbf{e}}$. The ion density and velocity gradients are set to zero at the boundaries, i.e., $\nabla n_{i}=0$ and $\nabla \cdot \mathbf{u}_{\mathbf{i}}=0$.

At the top dielectric surface, the boundary condition for the electric potential $\phi$ is given by ${ }^{27}$

$$
\phi=\frac{\phi_{1}+\Delta z\left(\sigma_{s} / \varepsilon_{0}+\varepsilon_{d} \phi_{2} / \varepsilon_{0} / L\right)}{1+\Delta z \varepsilon_{d} / \varepsilon_{0} / L} .
$$

Here, $\phi_{1}$ is the plasma potential at a distance $\Delta z$ from the dielectric surface, and $\phi_{2}$ is the potential on the opposite side of the dielectric from the plasma; $\sigma_{s}$ is the surface charge density; and $\varepsilon_{d}$ and $L$ are the permittivity and thickness of the dielectric region, respectively.

\section{Electromagnetic module}

In ICP discharges, the plasma is generated by the inductively coupled electric fields, which are governed by the Maxwell equations

$$
\begin{aligned}
& \nabla \times \mathbf{E}=-\frac{\partial \mathbf{B}}{\partial t}, \\
& \nabla \times \mathbf{B}=\mu_{0} \mathbf{J}+\mu_{0} \varepsilon_{0} \varepsilon_{r} \frac{\partial \mathbf{E}}{\partial t},
\end{aligned}
$$

where $\mathbf{E}$ and $\mathbf{B}$ are the time-varying electric field and magnetic field; $\mu_{0}$ and $\varepsilon_{r}$ are the vacuum permeability and the relative dielectric constant of the quartz window, respectively. The plasma current in the discharge region is given by

$$
\frac{\partial \mathbf{J}}{\partial t}=\frac{e^{2} n_{e}}{m_{e}} \mathbf{E}-\nu_{e n} \mathbf{J}
$$

However, in the vacuum region and dielectric region, the plasma current $\mathbf{J}$ equals zero.

Based on the boundary conditions for the electromagnetic fields at the material interface $\mathbf{n} \times\left(\mathbf{E}_{2}-\mathbf{E}_{1}\right)=0$ and $\mathbf{n} \times\left(\mathbf{B}_{2}-\mathbf{B}_{1}\right)=\mu_{0} \alpha$, both the azimuthal component of the electric field $E_{\theta}$ and the radial component of the magnetic field $B_{r}$ are continuous at the interface of the plasma region and the dielectric window. However, at the interface of the dielectric window and the vacuum region, where the coil set is placed, $\left.E_{\theta}\right|_{v a c}=\left.E_{\theta}\right|_{d i e}$ and $\left.B_{r}\right|_{v a c}-\left.B_{r}\right|_{d i e}=\mu_{0} \alpha_{\theta}$, where $\alpha_{\theta}$ is the current density.

\section{E. Sheath module}

In order to accurately investigate the bias effect on the plasma characteristics under various discharge conditions, a sheath module should be included in the MAPS-ICP solver. Over the past few years, the behavior of the sheath has been studied by various sheath models. ${ }^{28-35}$ In this paper, we assume that the sheath is uniform in the radial direction. This is justified because, although the electron density is not uniform in the radial direction, the absolute value in the sheath only has a $20 \%$ drop from the center to the radial edge. Moreover, only the axial components of the electron flux and the ion flux are needed in the current balance equation to solve for the potential at the electrode, as will be discussed below. Therefore, in order to reduce the computational burden, a self-consistent one-dimensional dynamic sheath model is integrated into the two-dimensional MAPS-ICP solver. More specifically, in the sheath, the spatiotemporal distributions of the charged particle density and flux, as well as of the electric potential, are obtained by solving equations (1) and 
(5)-(7) in the axial direction. The plasma properties at the bulk-sheath interface calculated in the inductive plasma module are used as the boundary conditions in the capacitive sheath module.

The instantaneous voltage on the substrate depends not only on the applied bias power but also on the transient behavior of the sheath. Therefore, an equivalent circuit model has been presented, ${ }^{33,35}$ in which the sheath is treated as a parallel combination of a diode, a capacitor, and a current source. Assuming that the current applied at the electrode from the bias power is sinusoidal, the current balance equation is given by

$$
I_{i}-I_{e}-I_{d}=I_{\max } \sin (2 \pi f t) .
$$

Here, $I_{\max }$ and $f$ are the amplitude and frequency of the bias current. $I_{i}=e\left(\sum Z_{+} n_{+} u_{+}-\sum Z_{-} n_{-} u_{-}\right) A$ is the ion current, $I_{e}=e n_{e} u_{e} A$ is the electron current at the electrode, and $A$ is the surface area of the electrode. The displacement current $I_{d}$ can be derived as

$$
I_{d}=C_{s} \frac{d V_{e}}{d t}+V_{e} \frac{d C_{s}}{d t},
$$

where $C_{s}=\varepsilon_{0} A / d_{s}$ is the sheath capacitance, and $d_{s}$ is the sheath thickness. The potential at the electrode $V_{e}$ is obtained as output of the above current balance equation and is used as the boundary condition in the inductive plasma module above, when solving the Poisson equation. From the current balance equation, it is clear that the potential at the electrode $V_{e}$ depends not only on the applied bias power but also on the behavior of the electrons and ions in the sheath, as well as the movement of the sheath. Therefore, the sheath dynamics has an influence on the potential at the electrode and accordingly affects the plasma properties in the bulk region. Based on the variation of $V_{e}$ as a function of time during one RF cycle, the bias voltage is self-consistently calculated.

\section{RESULTS AND DISCUSSION}

\section{A. Bias effect at different coil currents}

The influence of the bias effect on the discharge mode transition and plasma characteristics in an ICP reactor is investigated at different coil currents (i.e., 6, 11, and $13 \mathrm{~A}$ ). The simulations were performed at a pressure of $20 \mathrm{mTorr}$ at various bias voltages in the range of $0-300 \mathrm{~V}$. In the simulation, the secondary electron emission coefficient is assumed to be 0.1 . Since the collisionless heating and the stochastic heating caused by the biased sheath oscillation can be ignored when the pressure is not extremely low, the discharge is mainly sustained by the Ohmic heating. The frequencies of the coil set and of the bias power are fixed at $13.56 \mathrm{MHz}$.

The spatiotemporal averaged electron density and the ratio of the absorbed bias power $\mathrm{P}_{\text {bias }}$ to the total power $\mathrm{P}_{\text {total }}$ (i.e., the sum of the power absorption from the coil current and the bias source) are plotted in Fig. 2 as a function of bias voltage at different ICP currents. It is clear that when the ICP current is relatively low, i.e., $6 \mathrm{~A}$, the electron density
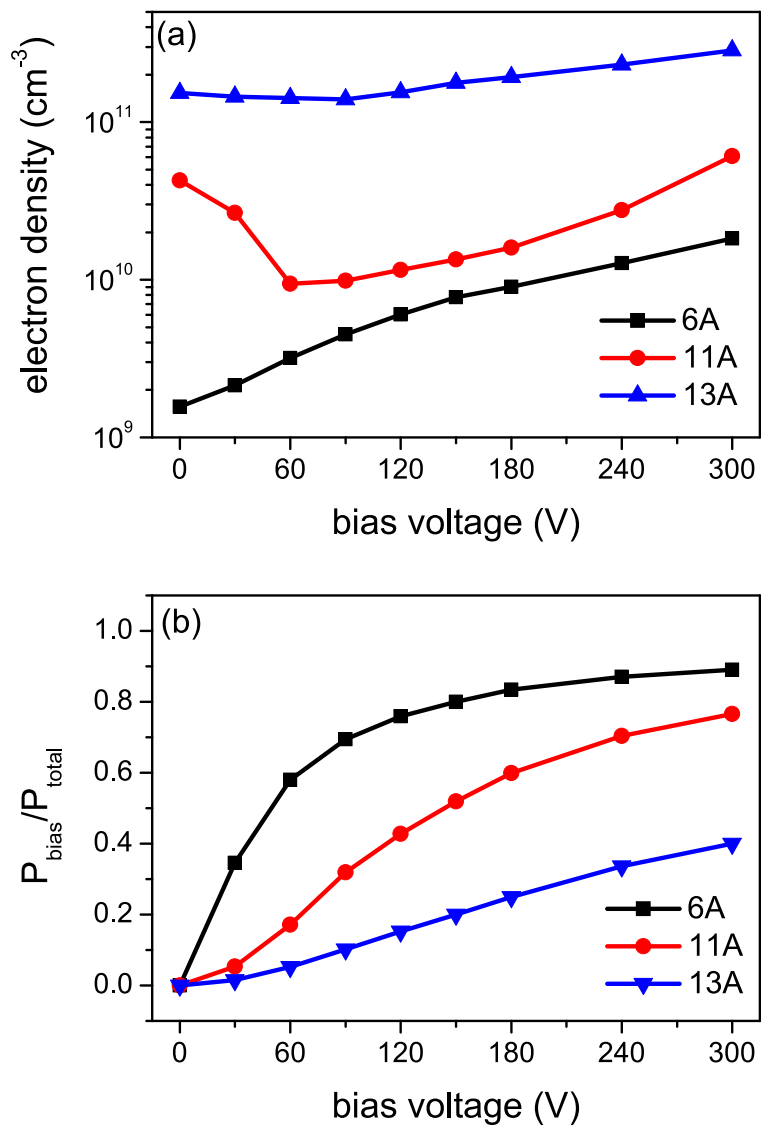

FIG. 2. (Color online) Evolutions of (a) the spatiotemporal averaged plasma density, and (b) the ratio of the bias power to the total power, with bias voltage at various ICP currents, for an argon discharge sustained at $13.56 \mathrm{MHz}$.

increases monotonically with bias voltage, which agrees well with experimental data. ${ }^{9,10}$ Indeed, at the low coil current, the bias power plays a dominate role in the plasma generation. The inductive power absorption from the coil current is about $11 \mathrm{~W}$ in the case without bias power, and it increases to $58 \mathrm{~W}$ at $300 \mathrm{~V}$, whereas the capacitive bias power is $477 \mathrm{~W}$ under this condition. The ratio of $\mathrm{P}_{\text {bias }}$ to $\mathrm{P}_{\text {total }}$ is indeed as high as $90 \%$ at the bias voltage of $300 \mathrm{~V}$. The absorbed bias power in the plasma increases significantly with bias voltage, and therefore results in a higher plasma density (i.e., around $1.6 \times 10^{9} \mathrm{~cm}^{-3}$ when there is no bias power and $1.8 \times 10^{10} \mathrm{~cm}^{-3}$ at the bias voltage of $300 \mathrm{~V})$.

However, when the ICP current becomes higher, i.e., 11 and $13 \mathrm{~A}$, the plasma density shows a strikingly different trend with rising bias voltage. At the ICP current of $11 \mathrm{~A}$, the plasma density first exhibits a rapid decrease with bias voltage (i.e., from $4.3 \times 10^{10} \mathrm{~cm}^{-3}$ at $0 \mathrm{~V}$ to $9.4 \times 10^{9} \mathrm{~cm}^{-3}$ at $60 \mathrm{~V})$, and subsequently, it increases to $6.1 \times 10^{10} \mathrm{~cm}^{-3}$ at $300 \mathrm{~V}$. Likewise, at an ICP current of $13 \mathrm{~A}$, the electron density decreases slightly from $1.5 \times 10^{11} \mathrm{~cm}^{-3}$ at $0 \mathrm{~V}$ bias voltage until $1.4 \times 10^{11} \mathrm{~cm}^{-3}$ at a bias voltage of $90 \mathrm{~V}$, and subsequently, it increases again to about $2.9 \times 10^{11} \mathrm{~cm}^{-3}$ at $300 \mathrm{~V}$ bias voltage. This behavior is similar to that observed in Ref. 13. Indeed, as the current in the coil set increases, especially at $13 \mathrm{~A}$, the discharge is mainly sustained by the 
inductive field. This can also be understood by examining the ratio between $\mathrm{P}_{\text {bias }}$ and $\mathrm{P}_{\text {total }}$ in Fig. 2(b). At $13 \mathrm{~A}$, although the bias power is $501 \mathrm{~W}$ at $300 \mathrm{~V}$, the inductive power absorption increases from $499 \mathrm{~W}$ in the case without bias source to $752 \mathrm{~W}$ at $300 \mathrm{~V}$, and therefore, the ratio is lower than 0.4 at all selected bias voltages with a fixed ICP current of $13 \mathrm{~A}$.

The initial drop in electron density when applying a bias voltage in the case of 11 or 13 A ICP coil current can be understood by considering a global model ${ }^{1,10}$

$$
n_{0}=\frac{P_{\text {total }}}{e u_{B}\left(A_{\mathrm{eff}, g} \varepsilon_{T, g}+A_{\mathrm{eff}, s} \varepsilon_{T, s}\right)} .
$$

Here, $P_{\text {total }}$ is the total power absorption, $u_{B}$ is the Bohm velocity, $A_{\mathrm{eff}, g}, A_{\mathrm{eff}, s}, \varepsilon_{T, g}$, and $\varepsilon_{T, s}$ are the effective area for particle loss and the total energy loss at the grounded wall and the substrate, respectively. The total energy $\operatorname{loss} \varepsilon_{T}$ consists of three parts: the collisional energy loss $\varepsilon_{c}$, the kinetic energy loss per electron $\varepsilon_{e}$, and per ion $\varepsilon_{i}$. In our simulations, the electron temperature shows little change with the bias voltage, and so are $\varepsilon_{c}$ and $\varepsilon_{e}{ }^{1}$ Since $\varepsilon_{i}$ is the sum of the ion energy entering the sheath and the energy gained by the ion as it traverses the sheath, $\varepsilon_{i}$ at the substrate depends strongly on the sheath voltage and is much higher than that at the grounded wall. Therefore, the plasma density is approximately proportional to the ratio of the total power absorption $P_{\text {total }}$ to the kinetic energy lost per ion $\varepsilon_{i}$. When a small bias voltage is applied, although the total power deposition increases with bias voltage, the bias power dissipation caused by the ion acceleration is more dominant, ${ }^{10,13}$ and this gives rise to a considerable decrease in the plasma density. As the bias voltage increases further, the electrons gain more energy from the higher capacitive bias power, and therefore, these energetic electrons enhance the plasma generation.

The bias effect on the discharge mode transition and on the plasma characteristics at the ICP current of $6 \mathrm{~A}$ is apparent from the spatial distributions of the plasma density in Fig. 3. When there is no bias power applied on the substrate [Fig. 3(a)], the discharge is sustained by the pure inductive power, and the peak of the plasma density appears along the reactor centerline $(\mathrm{z}=8.5 \mathrm{~cm})$. As the bias voltage increases to $90 \mathrm{~V}\left(\mathrm{P}_{\text {bias }}=47 \mathrm{~W}\right)$, no significant effect on the density is detected, except for the absolute values, which are nearly 3.5 times as high as obtained without bias power. At the bias voltage of $300 \mathrm{~V}\left(\mathrm{P}_{\text {bias }}=477 \mathrm{~W}\right)$, as shown in Fig. 3(c), the maximum of the plasma density moves slightly toward the bottom electrode with higher absolute values. This indicates that the bias power has a dominant influence on the plasma characteristics under this condition, and the electrons gain more energy from the higher capacitive bias power, enhancing the ionization.

In order to understand the different plasma density profiles, the spatial distributions of the ionization rate obtained at various voltages are presented in Fig. 4. When the ICP current is low (i.e., $6 \mathrm{~A}$ ) without RF bias power [Fig. 4(a)], the electrons are heated mainly by the inductive azimuthal electric field
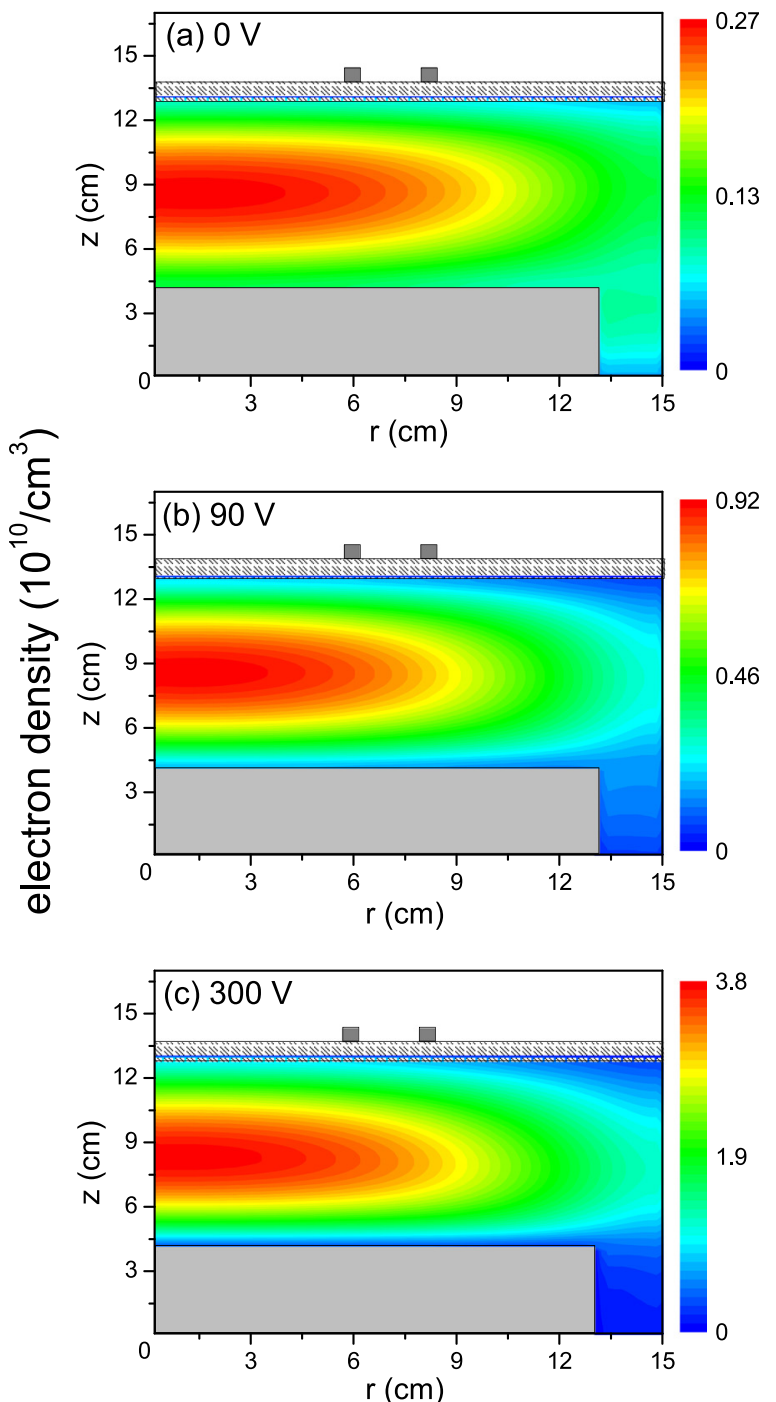

FIG. 3. (Color online) Distributions of the plasma density at different bias voltages: (a) $0 \mathrm{~V}$, (b) $90 \mathrm{~V}$, and (c) $300 \mathrm{~V}$, for an argon discharge sustained at an ICP current of $6 \mathrm{~A}$ and $13.56 \mathrm{MHz}$, with a bias frequency of 13.56 $\mathrm{MHz}$.

induced by the coil current, and the maximum of the ionization rate appears at the reactor center and close to the dielectric window. The absolute value is very low, i.e., in the order of $1.0 \times 10^{14} \mathrm{~cm}^{-3} \mathrm{~s}^{-1}$. As the bias voltage increases to $90 \mathrm{~V}$, the electrons are heated not only by the inductive field but also by the capacitive field caused by the RF bias. Therefore, the electrons gain more energy from the bias power deposition, which gives rise to a considerable increase in the plasma generation (leading to an ionization rate of $4.6 \times 10^{14} \mathrm{~cm}^{-3}$ $\mathrm{s}^{-1}$ ), and the maximum of the ionization rate moves toward the bottom electrode, as is clear from Fig. 4(b). Finally, when a significant bias power is applied, corresponding to $300 \mathrm{~V}$ [Fig. 4(c)], the discharge mode exhibits a transition. Indeed, the discharge is mainly sustained by the capacitive bias power, and accordingly, a prominent peak in the ionization rate is observed along $\mathrm{z}=8.5 \mathrm{~cm}$, moving closer to the bottom electrode at about $r=9 \mathrm{~cm}$. Furthermore, the absolute value is one order of magnitude higher than that obtained in the case without bias voltage. 

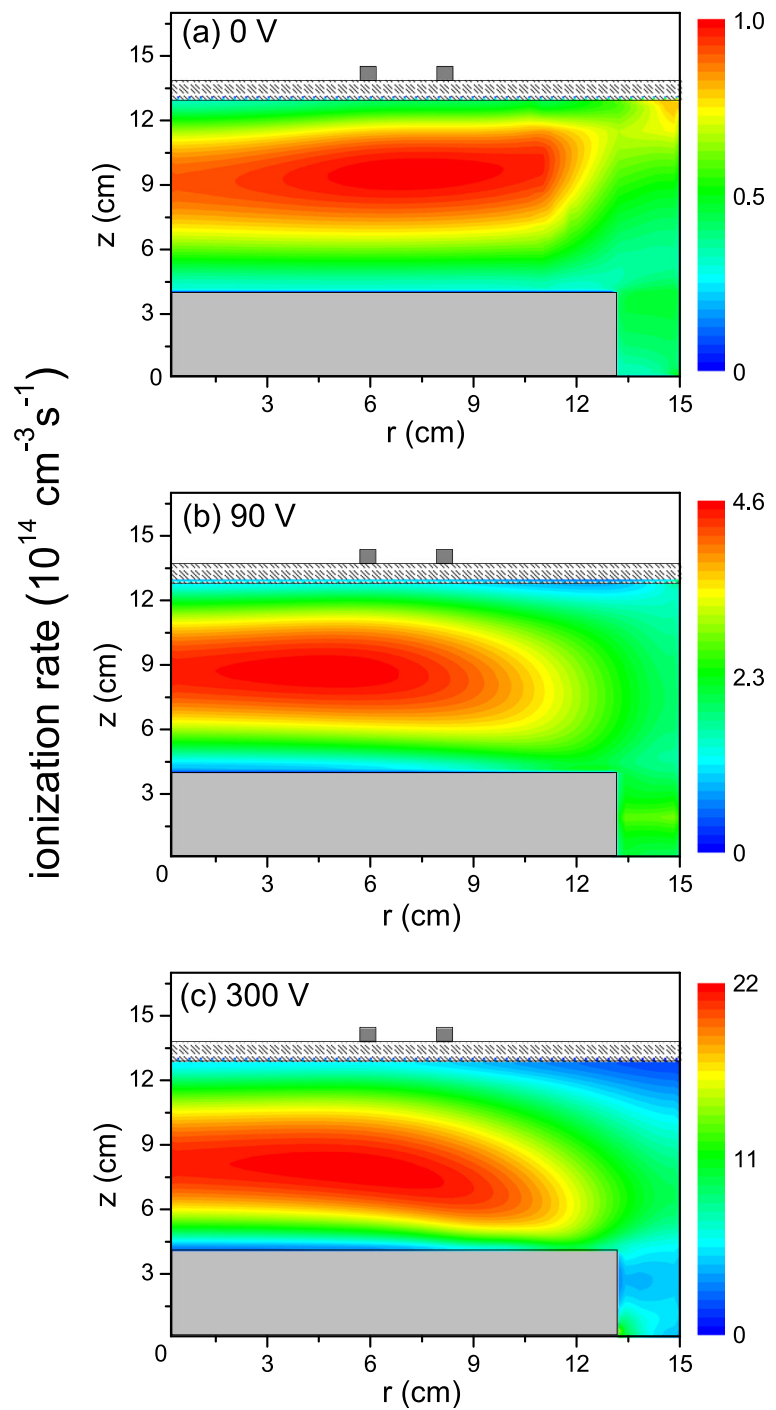

FIG. 4. (Color online) Distributions of the ionization rate at different bias voltages: (a) $0 \mathrm{~V}$, (b) $90 \mathrm{~V}$, and (c) $300 \mathrm{~V}$, for an argon discharge sustained at an ICP current of $6 \mathrm{~A}$ and $13.56 \mathrm{MHz}$, with a bias frequency of 13.56 $\mathrm{MHz}$.

When the ICP current increases to 11 and $13 \mathrm{~A}$, the maximum of the plasma density becomes more intense and localized at the reactor center at all selected bias voltages, and the bias power has no significant influence on the distribution of the plasma density, except for the absolute values (not shown here). This is because the inductive coil power has a dominant influence on the plasma characteristics, especially at $13 \mathrm{~A}$.

When the ICP current increases to $11 \mathrm{~A}$, the spatial distributions of the ionization rate at various bias voltages are strikingly different from those obtained at $6 \mathrm{~A}$, both in shape and especially in absolute values. When the bias voltage is equal to 0, shown in Fig. 5(a), the ionization rate is characterized by a remarkable peak at the reactor center near the dielectric window. By comparing this with the results obtained at the ICP current of $6 \mathrm{~A}$, the ionization rate is more localized with a higher absolute value, i.e., at a maximum about $4.5 \times 10^{15} \mathrm{~cm}^{-3} \mathrm{~s}^{-1}$. As the bias voltage increases to $90 \mathrm{~V}\left[\mathrm{P}_{\text {bias }}=57 \mathrm{~W}\right.$, see Fig. 5(b)], the RF bias power starts to
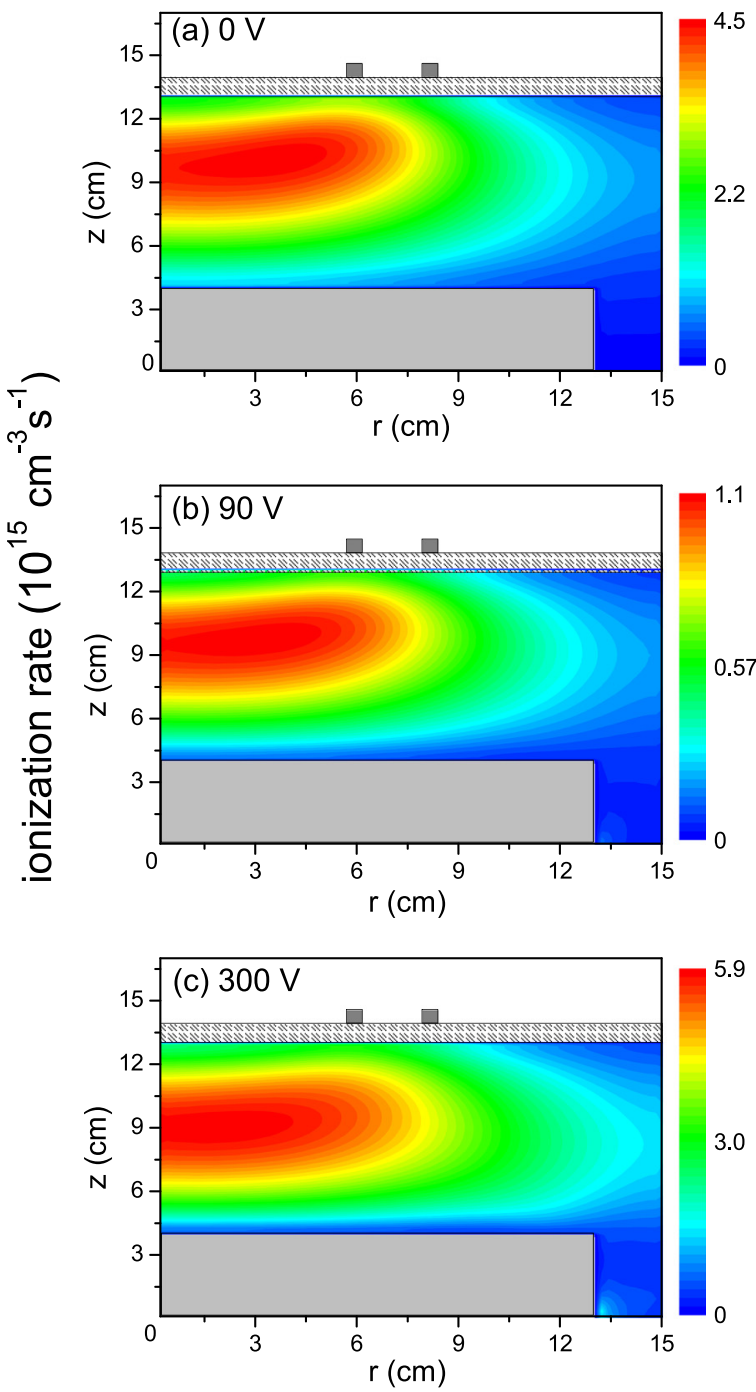

FIG. 5. (Color online) Distributions of the ionization rate at different bias voltages: (a) $0 \mathrm{~V}$, (b) $90 \mathrm{~V}$, and (c) $300 \mathrm{~V}$, for an argon discharge sustained at an ICP current of $11 \mathrm{~A}$ and $13.56 \mathrm{MHz}$, with a bias frequency of 13.56 $\mathrm{MHz}$.

have an influence on the plasma properties, and the maximum of the ionization rate moves away from the dielectric window, which is similar to the result obtained in the low current case [Fig. 4(b)]. As explained above, since more power dissipation occurs due to the ion acceleration, the ionization rate dramatically decreases to values of $1.1 \times 10^{15} \mathrm{~cm}^{-3} \mathrm{~s}^{-1}$. When the bias voltage increases further to $300 \mathrm{~V}\left(\mathrm{P}_{\text {bias }}=492 \mathrm{~W}\right)$, the peak of the ionization rate appears almost along the reactor centerline $(z=9 \mathrm{~cm})$, and the absolute value becomes higher again. Although more power is dissipated due to the ion acceleration as the bias voltage increases, the capacitive power deposition induced by the RF bias has a predominant influence on the plasma production when the bias voltage is as high as $300 \mathrm{~V}$.

The evolution of ionization rate profiles with increasing bias voltage at the ICP current of $13 \mathrm{~A}$ is depicted in Fig. 6. It is clear that the ionization rate is localized at the reactor center near the quartz window at all selected bias voltages, which indicates that the RF bias power has no profound 

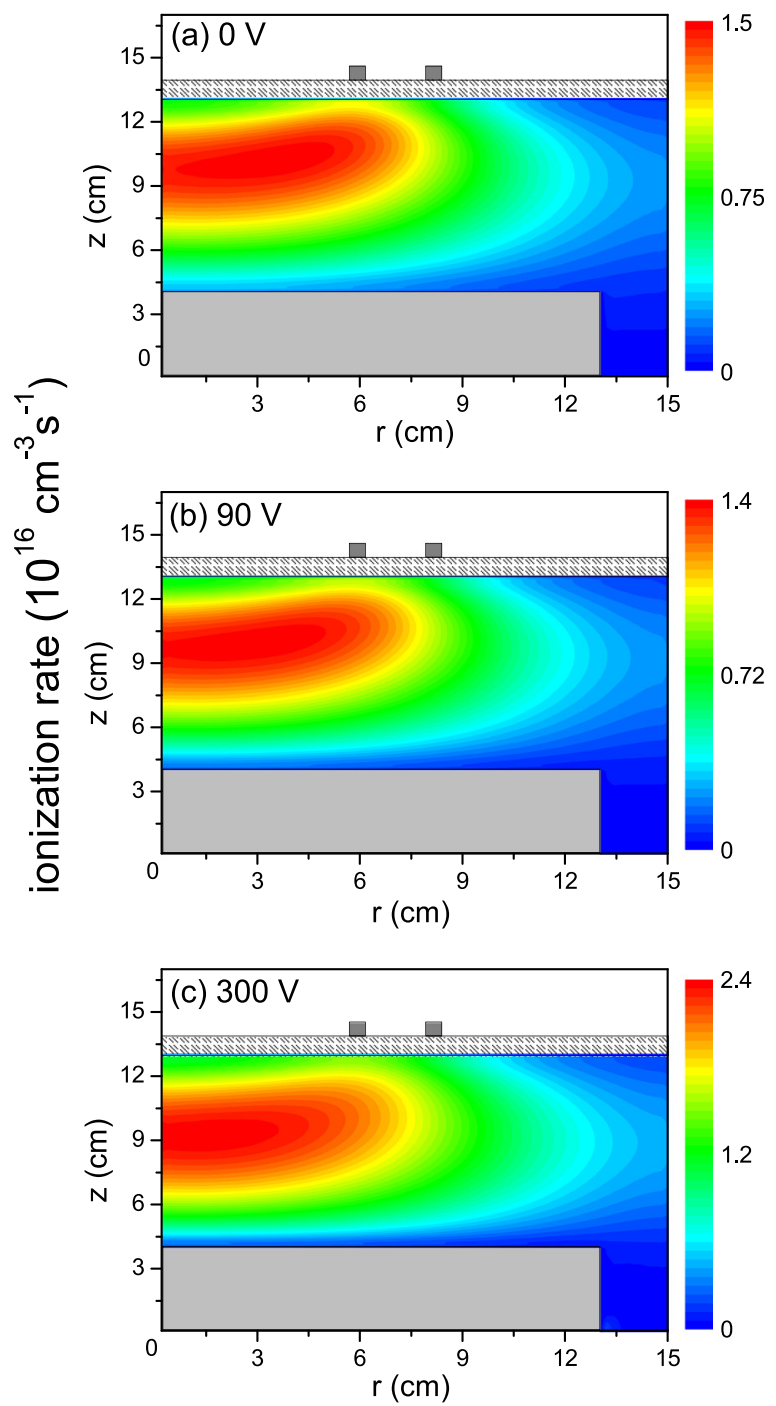

FIG. 6. (Color online) Distributions of the ionization rate at different bias voltages: (a) $0 \mathrm{~V}$, (b) $90 \mathrm{~V}$, and (c) $300 \mathrm{~V}$, for an argon discharge sustained at an ICP current of $13 \mathrm{~A}$ and $13.56 \mathrm{MHz}$, with a bias frequency of 13.56 $\mathrm{MHz}$.

influence on the plasma characteristics under these conditions. This is because when the ICP current is relatively high, the inductive field contributes significantly to the plasma generation and is responsible for the spatial distribution of the ionization rate. In addition, the ionization rate first slightly decreases, and then increases with rising bias voltage. This can again be explained by the competing mechanisms between the power dissipation due to the ion acceleration and the power absorption induced by the high bias power.

To visualize the different bias effects at various ICP currents, the profiles of the ionization rate at $r=7 \mathrm{~cm}$ (i.e., in the middle of the two-turns planar coil) are plotted in Figs. 7-9 as a function of axial position and time within $1 \mathrm{RF}$ cycle (1 RF period $=74 \mathrm{~ns}$ ). In a pure inductive discharge without RF bias source and at the coil current of 6 A [Fig. 7(a)], the electrons are heated by the inductive field arising from the ICP current, which is reflected by a substantial ionization in the bulk region with two maxima during one period. Indeed, the
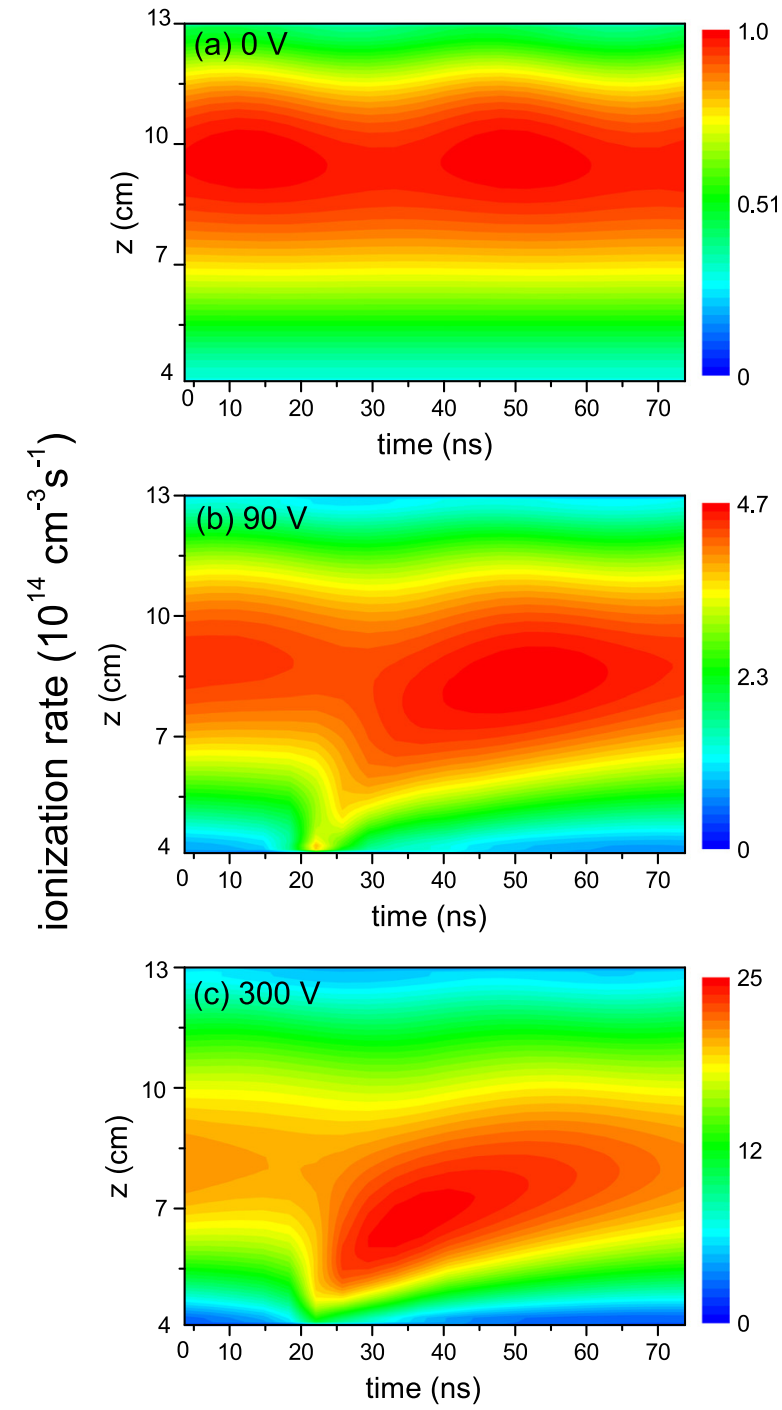

FIG. 7. (Color online) Spatiotemporal distributions of the ionization rate at $r$ $=7 \mathrm{~cm}$ at different bias voltages: (a) $0 \mathrm{~V}$, (b) $90 \mathrm{~V}$, and (c) $300 \mathrm{~V}$, for an argon discharge sustained at an ICP current of $6 \mathrm{~A}$ and $13.56 \mathrm{MHz}$, with a bias frequency of $13.56 \mathrm{MHz}$.

inductive field is first quite strong, therefore heating and accelerating the electrons, which results in a large amount of ionization (with a rate around $1.0 \times 10^{14} \mathrm{~cm}^{-3} \mathrm{~s}^{-1}$ ). At a later time, the inductive field becomes weaker, but then it reverses, and hence produces a second peak of the same magnitude. Moreover, the ionization rate near the bottom electrode is constant in time with a relatively low value, i.e., in the order of $2.5 \times 10^{13} \mathrm{~cm}^{-3} \mathrm{~s}^{-1}$. By switching on the bias source at $90 \mathrm{~V}$ [see Fig. 7(b)], the maximum of the ionization rate moves downwards, explaining the result shown in Figs. 3(b) and 4(b). Besides, the ionization rate near the bottom electrode becomes much more pronounced (i.e., around $2.0 \times 10^{14} \mathrm{~cm}^{-3}$ $\mathrm{s}^{-1}$ ), and it exhibits some fluctuation as a function of time. This means that the influence of the capacitive power becomes remarkable, and the discharge mode transition occurs. When the bias voltage is equal to $300 \mathrm{~V}$, only one peak per RF cycle is observed instead of two, which appears near the bottom electrode, and the maximum ionization rate is much higher (i.e., almost $2.5 \times 10^{15} \mathrm{~cm}^{-3} \mathrm{~s}^{-1}$ ). By comparing 

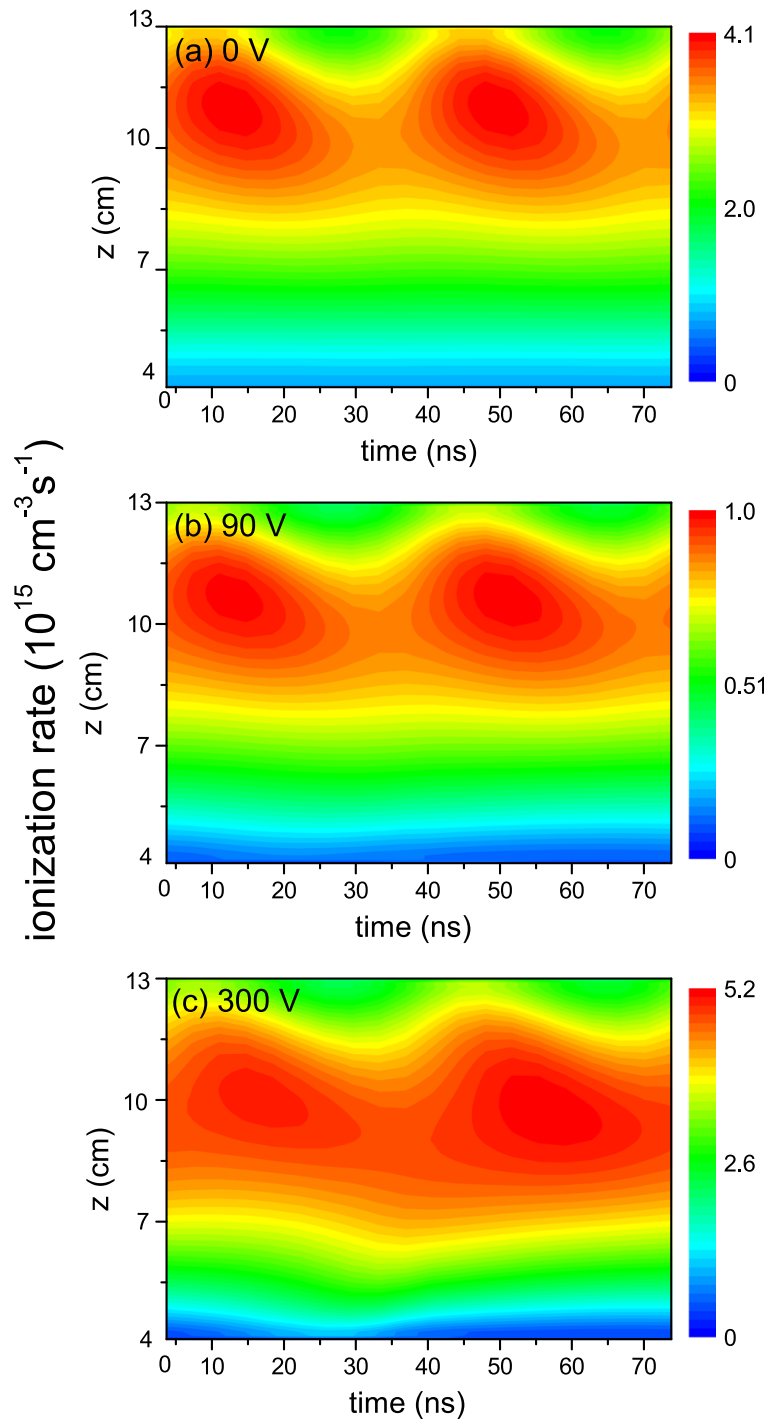

FIG. 8. (Color online) Spatiotemporal distributions of the ionization rate at $r$ $=7 \mathrm{~cm}$ at different bias voltages: (a) $0 \mathrm{~V}$, (b) $90 \mathrm{~V}$, and (c) $300 \mathrm{~V}$, for an argon discharge sustained at an ICP current of $11 \mathrm{~A}$ and $13.56 \mathrm{MHz}$, with a bias frequency of $13.56 \mathrm{MHz}$.

with the ionization rate calculated without bias power, the discharge is mainly sustained by the RF bias power under this condition. A similar spatiotemporal distribution of the electron impact excitation rate has also been observed in neon plasmas. $^{11}$

The spatiotemporal distributions of the ionization rate at different bias voltages with a fixed ICP current of $11 \mathrm{~A}$ are presented in Fig. 8. When the bias source is switched off, the ionization rate displays two peaks under the dielectric window during one period, as is observed from Fig. 8(a). It is clear that the peaks are more intense than those illustrated in Fig. 7(a) (i.e., about $4.1 \times 10^{15} \mathrm{~cm}^{-3} \mathrm{~s}^{-1}$ versus about $1.0 \times 10^{14} \mathrm{~cm}^{-3} \mathrm{~s}^{-1}$ ), because the heating is more localized at higher coil current. By switching on the bias source, a clear difference can be observed, which indicates that the bias power plays an important role in the plasma behavior. At the bias voltage of $90 \mathrm{~V}$, the two peaks become less prominent with much lower values (i.e., around $1.0 \times 10^{15} \mathrm{~cm}^{-3} \mathrm{~s}^{-1}$ ),
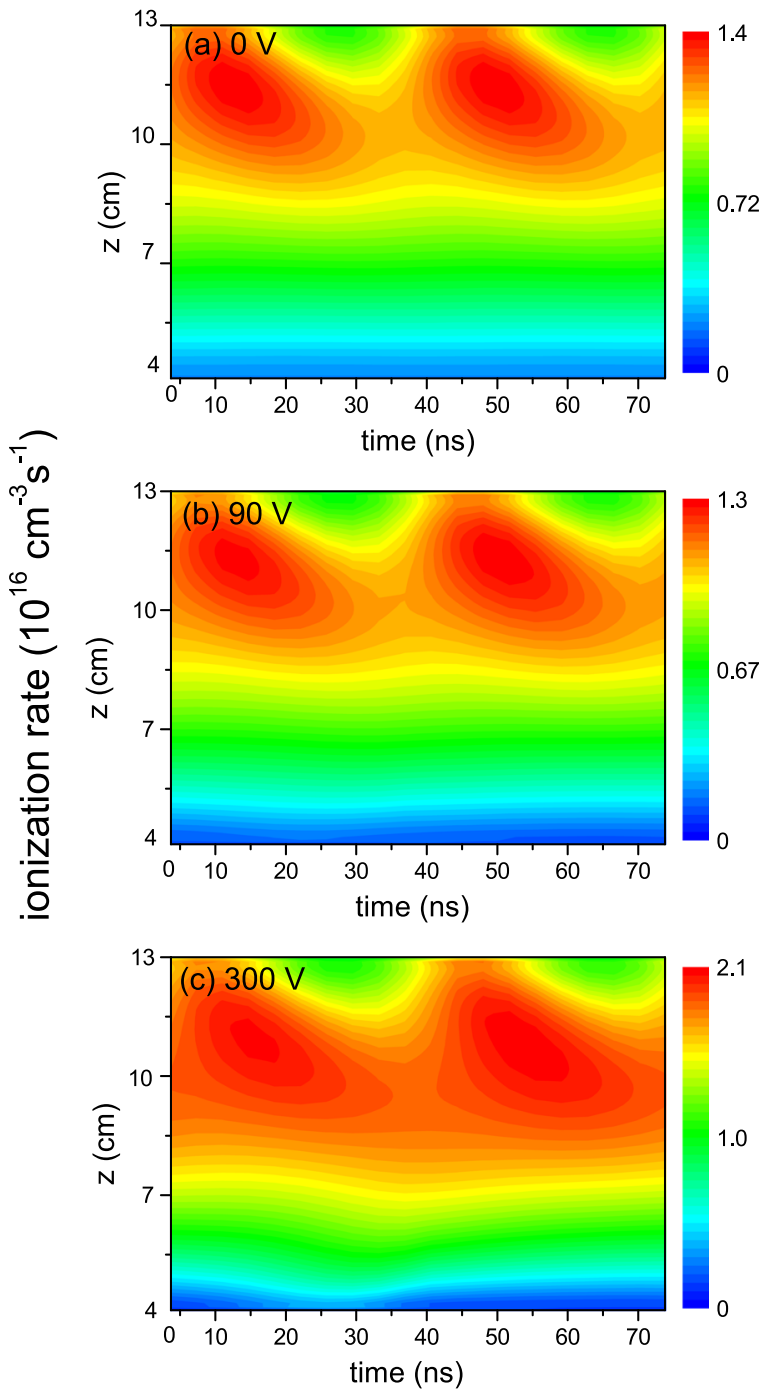

FIG. 9. (Color online) Spatiotemporal distributions of the ionization rate at $r$ $=7 \mathrm{~cm}$ at different bias voltages: (a) $0 \mathrm{~V}$, (b) $90 \mathrm{~V}$, and (c) $300 \mathrm{~V}$, for an argon discharge sustained at an ICP current of $13 \mathrm{~A}$ and $13.56 \mathrm{MHz}$, with a bias frequency of $13.56 \mathrm{MHz}$.

explaining the density decrease shown in Fig. 2(a). When the bias voltage is as high as $300 \mathrm{~V}$, the ionization rate becomes higher again (i.e., $5.2 \times 10^{15} \mathrm{~cm}^{-3} \mathrm{~s}^{-1}$ ), and the peaks move further downwards and appear at $\mathrm{z}=10 \mathrm{~cm}$, which is responsible for the ionization rate profile with higher absolute values in Fig. 5(c). Furthermore, the peak in the second half of the period is more intense than that in the first half of the period. This implies that the capacitive field has a significant influence on the plasma production in this case. Besides, a minimum in the ionization rate is detected near the biased electrode, and this can again be attributed to the modulation of the bias power.

Finally, when the coil current is as high as $13 \mathrm{~A}$ (cf. Fig. 9), the ionization rate is characterized by two peaks during one period at all selected bias voltages, which gives rise to a quite similar spatial distribution of the time-averaged ionization rate in Fig. 6. By comparing with the results obtained at lower ICP currents, it is clear that the maxima of the ionization rate are more intense (around $1.3 \times 10^{16} \mathrm{~cm}^{-3}$ 
$\mathrm{s}^{-1}$ or higher) and more localized near the quartz window, demonstrating that the inductive field plays a dominant role under this condition. When there is no bias power, the ionization rate near the bottom electrode is constant in time, as also observed in Fig. 8(a). As the bias voltage increases, shown in Figs. 9(b) and 9(c), the ionization rate near the bottom electrode exhibits some fluctuation as a function of time, especially at the bias voltage of $300 \mathrm{~V}\left(\mathrm{P}_{\text {bias }}=501 \mathrm{~W}\right)$. This is again because the capacitive field acts in the opposite direction at a later time within one RF cycle.

\section{B. Bias effect at different bias frequencies}

The bias effect on the discharge mode transition and plasma characteristics does not only depend on the ICP current, but also on the bias frequency. Therefore, in order to completely understand the bias effect, the spatiotemporal averaged plasma density, the ratio of $\mathrm{P}_{\text {bias }}$ to $\mathrm{P}_{\text {total }}$, as well as the distribution of the ionization rate are also calculated at different bias frequencies (i.e., 2, 13.56, and 27.12 MHz), with the ICP current fixed at $11 \mathrm{~A}$. Under this condition, the inductive power absorption is almost the same at different bias frequencies, i.e., about $139 \mathrm{~W}$ at $0 \mathrm{~V}$ bias voltage, and $150 \mathrm{~W}$ at $300 \mathrm{~V}$ bias voltage.

It is clear from Fig. 10(a) that the spatiotemporal averaged plasma density decreases first, and then increases with
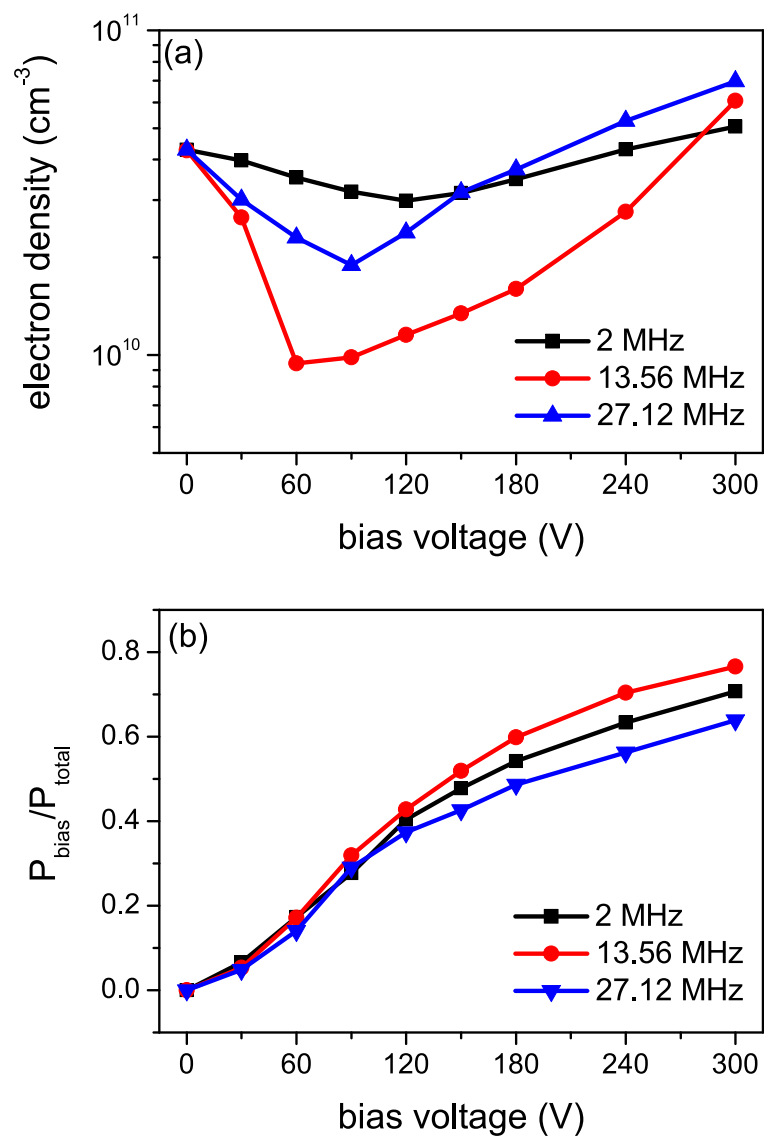

FIG. 10. (Color online) Evolutions of (a) the spatiotemporal averaged plasma density, and (b) the ratio of the bias power to the total power, with bias voltage at various bias frequencies, for an argon discharge sustained at an ICP current of $11 \mathrm{~A}$ and $13.56 \mathrm{MHz}$. rising bias voltage at all bias frequencies investigated, but the minima appear at different bias voltages. Indeed, when the bias frequency is $2 \mathrm{MHz}$, the minimum value appears at a bias voltage of $120 \mathrm{~V}$, whereas at $13.56 \mathrm{MHz}$ and $27.12 \mathrm{MHz}$, the minima take place at a bias voltage of 60 and $90 \mathrm{~V}$, respectively. This may be because when the bias power and the coil current are at the same frequency, the coupling efficiency of the two powers is higher than in the other cases. Furthermore, at $2 \mathrm{MHz}$, the plasma density exhibits only a slight increase with bias voltage (i.e., from $3.0 \times 10^{10}$ to $5.1 \times 10^{10} \mathrm{~cm}^{-3}$ at $2 \mathrm{MHz}$ ), whereas a more pronounced increase is observed at 13.56 and $27.12 \mathrm{MHz}$ (i.e., from $9.4 \times 10^{9}$ to $6.1 \times 10^{10} \mathrm{~cm}^{-3}$ at $13.56 \mathrm{MHz}$, and from $1.9 \times 10^{10}$ to $7.0 \times 10^{10} \mathrm{~cm}^{-3}$ at $27.12 \mathrm{MHz}$ ). This indicates that the bias has a more significant influence on the plasma characteristics at higher bias frequency. This is because electrons gain more energy from the more frequent collisions, and accordingly, they enhance the plasma generation. Besides, as the bias voltage increases, the ratio of $\mathrm{P}_{\text {bias }}$
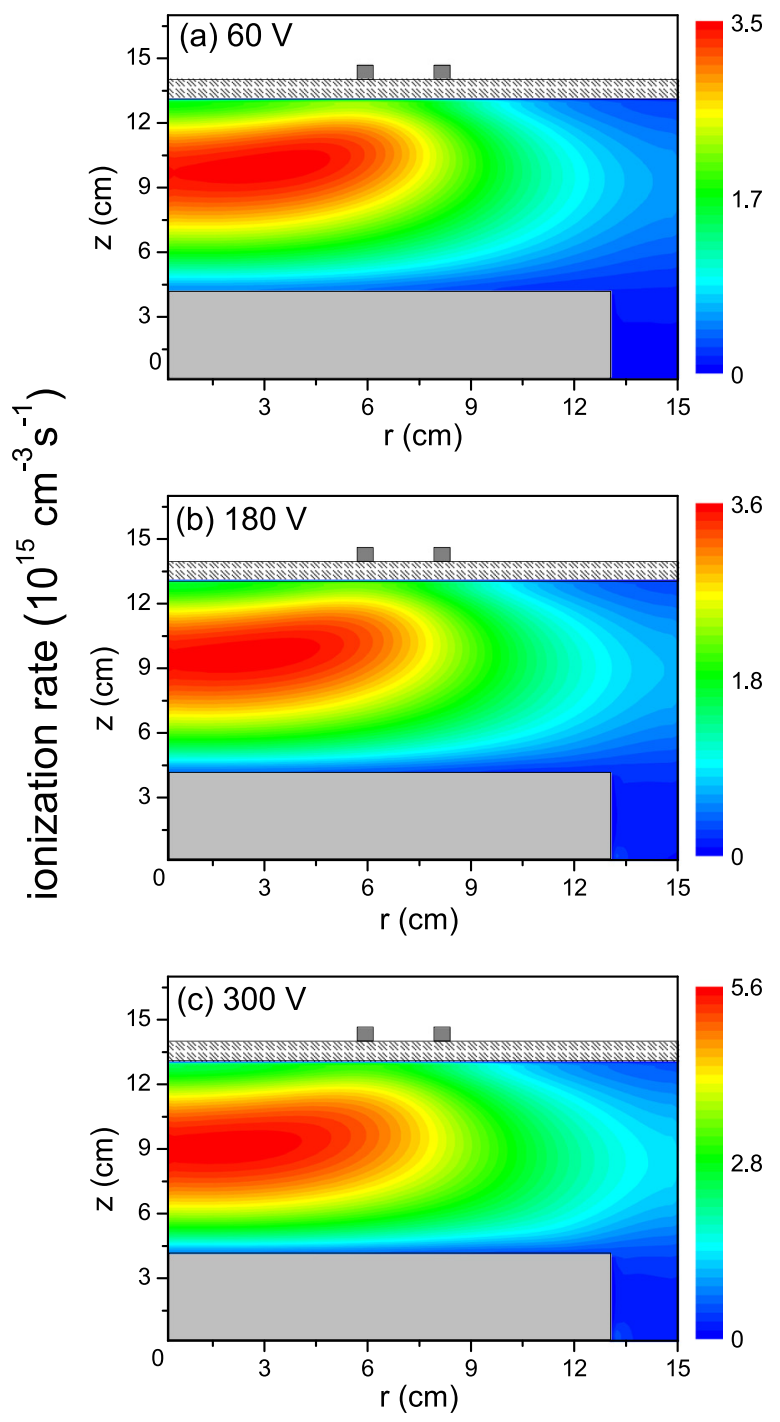

FIG. 11. (Color online) Distributions of the ionization rate at different bias voltages: (a) $60 \mathrm{~V}$, (b) $180 \mathrm{~V}$, and (c) $300 \mathrm{~V}$, for an argon discharge sustained at an ICP current of $11 \mathrm{~A}$ and $13.56 \mathrm{MHz}$, with a bias frequency of 2 $\mathrm{MHz}$. 
to $\mathrm{P}_{\text {total }}$ increases significantly at all bias frequencies, but the contribution of the bias power is somewhat lower at 27.12 MHz [cf. Fig. 10(b)]. This is again because of the lower coupling efficiency of the two powers.

The spatial distributions of the ionization rate at different bias voltages and a bias frequency of $2 \mathrm{MHz}$ are plotted in Fig. 11. When the bias electrode is grounded, the ionization rate (not shown here) is the same as in Fig. 5(a), which is characterized by a maximum at the center near the dielectric window. When the bias source is switched on with a voltage of $60 \mathrm{~V}\left(\mathrm{P}_{\text {bias }}=34 \mathrm{~W}\right)$ and $180 \mathrm{~V}\left(\mathrm{P}_{\text {bias }}=176 \mathrm{~W}\right)$, no significant effect on the ionization rate is detected, except for the lower absolute values (i.e., $3.5 \times 10^{15} \mathrm{~cm}^{-3} \mathrm{~s}^{-1}$ at $60 \mathrm{~V}$, and $3.6 \times 10^{15} \mathrm{~cm}^{-3} \mathrm{~s}^{-1}$ at $\left.180 \mathrm{~V}\right)$. As the bias voltage increases to $300 \mathrm{~V}\left(\mathrm{P}_{\text {bias }}=334 \mathrm{~W}\right)$, the ionization rate becomes more intense at the reactor center with higher absolute values, i.e., $5.6 \times 10^{15} \mathrm{~cm}^{-3} \mathrm{~s}^{-1}$, as shown in Fig. 11(c).

The effects of the bias power on the plasma characteristics at a bias frequency of $27.12 \mathrm{MHz}$ can be illustrated by
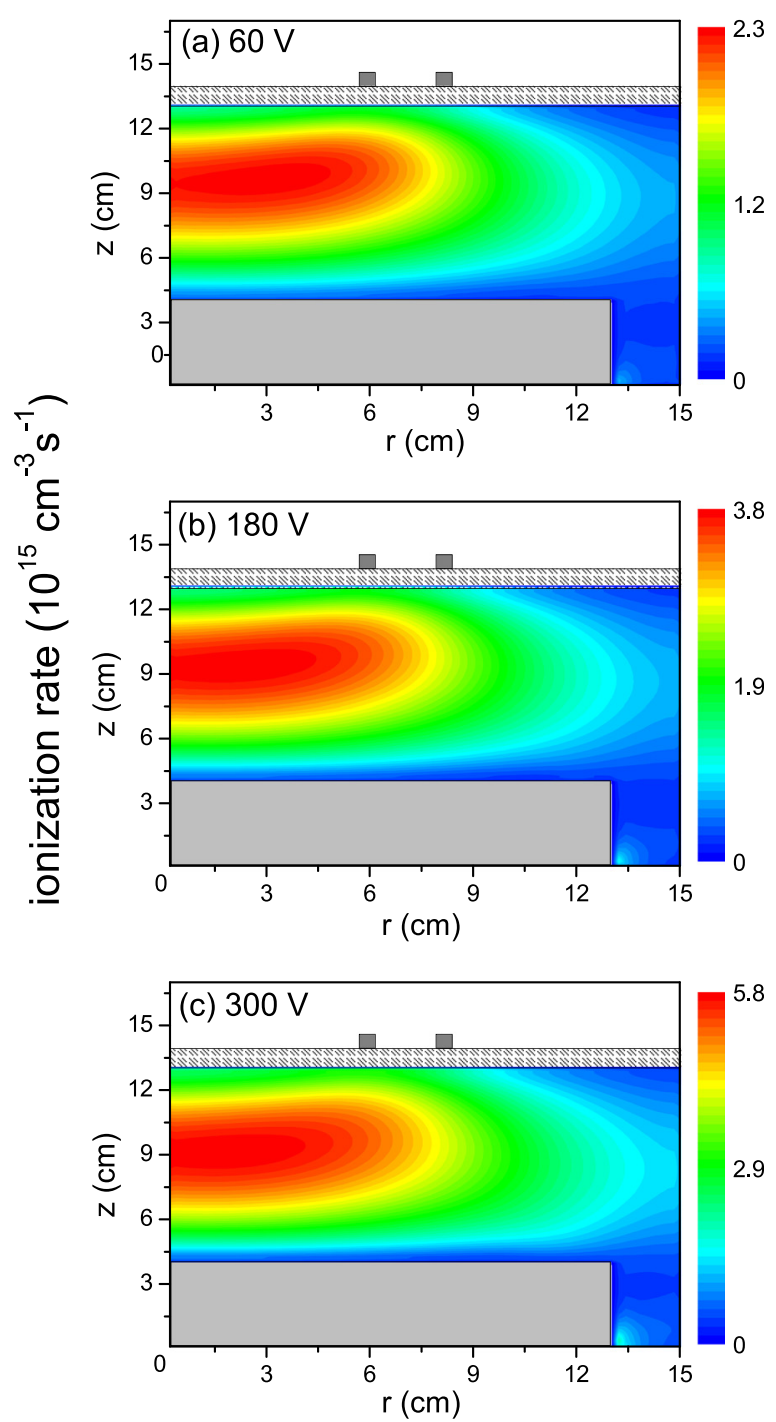

FIG. 12. (Color online) Distributions of the ionization rate at different bias voltages: (a) $60 \mathrm{~V}$, (b) $180 \mathrm{~V}$, and (c) $300 \mathrm{~V}$, for an argon discharge sustained at an ICP current of $11 \mathrm{~A}$ and $13.56 \mathrm{MHz}$, with a bias frequency of 27.12 MHz. the ionization rate distributions depicted in Fig. 12. By comparing with the results obtained at low bias frequency, the maxima of the ionization rate at $2 \mathrm{MHz}$ are closer to the dielectric window at all selected bias voltages. It is clear from Fig. 12(a) that the maximum of the ionization rate appears near the quartz window at about $\mathrm{z}=10 \mathrm{~cm}$ when the bias voltage is $60 \mathrm{~V}\left(\mathrm{P}_{\text {bias }}=23 \mathrm{~W}\right)$. As the bias voltage increases to $180 \mathrm{~V}$ and $300 \mathrm{~V}\left[\mathrm{P}_{\text {bias }}=136\right.$ and $278 \mathrm{~W}$, see Figs. 12(b) and 12(c)], the maximum moves toward the bottom electrode and appears at about $\mathrm{z}=9 \mathrm{~cm}$ at $300 \mathrm{~V}$. Moreover, the ionization rate increases with the bias voltage, and the absolute value, i.e., $5.8 \times 10^{15} \mathrm{~cm}^{-3} \mathrm{~s}^{-1}$ at $300 \mathrm{~V}$, is a factor of 2.5 higher than that obtained at $60 \mathrm{~V}$ (around $\left.2.3 \times 10^{15} \mathrm{~cm}^{-3} \mathrm{~s}^{-1}\right)$.

In order to understand the bias effect on the plasma transient behavior, the spatiotemporal distributions of the ionization rate obtained at various voltages are presented for a bias frequency of $2 \mathrm{MHz}$ and $27.12 \mathrm{MHz}$ in Figs. 13 and 14,
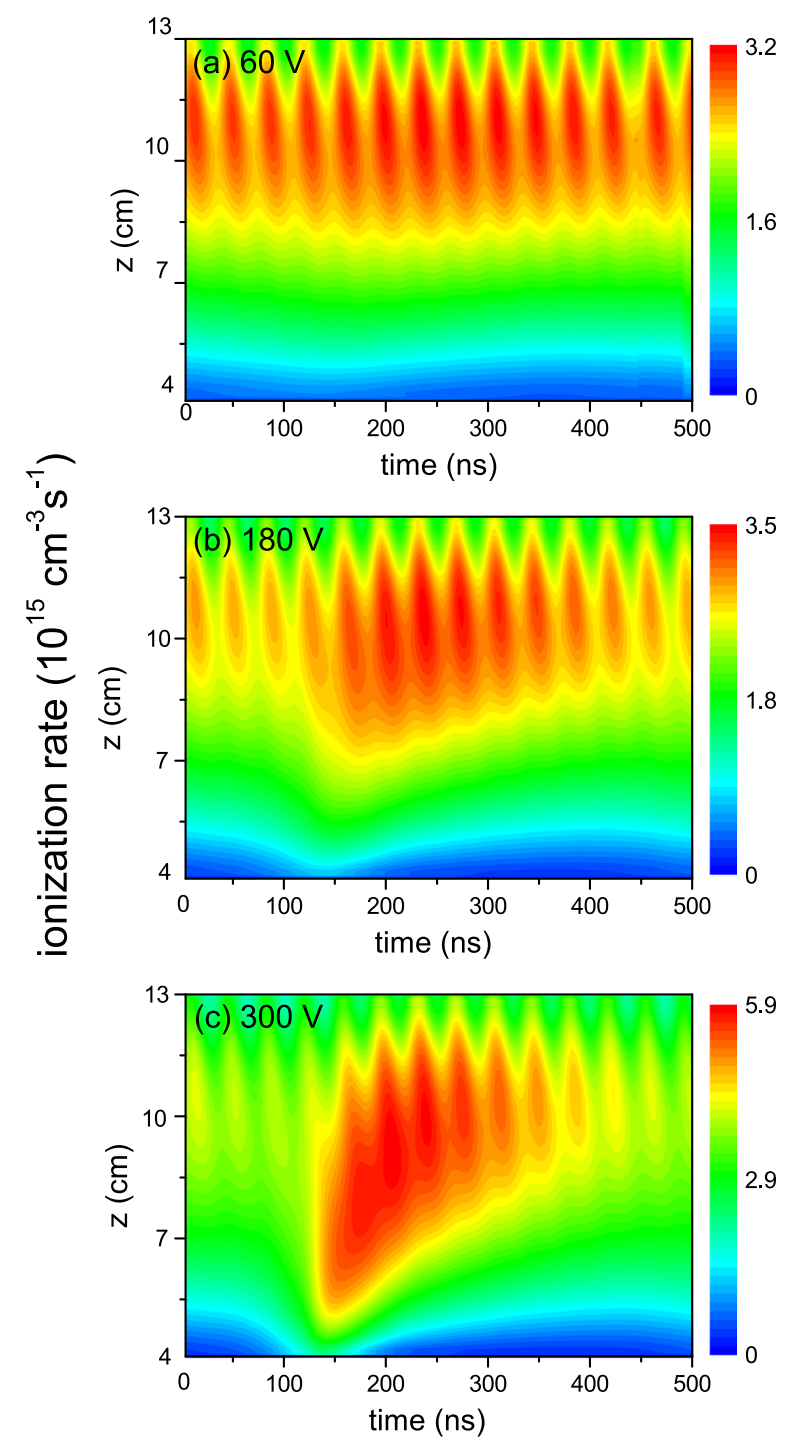

FIG. 13. (Color online) Spatiotemporal distributions of the ionization rate at $\mathrm{r}=7 \mathrm{~cm}$ at different bias voltages: (a) $60 \mathrm{~V}$, (b) $180 \mathrm{~V}$, and (c) $300 \mathrm{~V}$, for an argon discharge sustained at an ICP current of $11 \mathrm{~A}$ and $13.56 \mathrm{MHz}$, with a bias frequency of $2 \mathrm{MHz}$. 


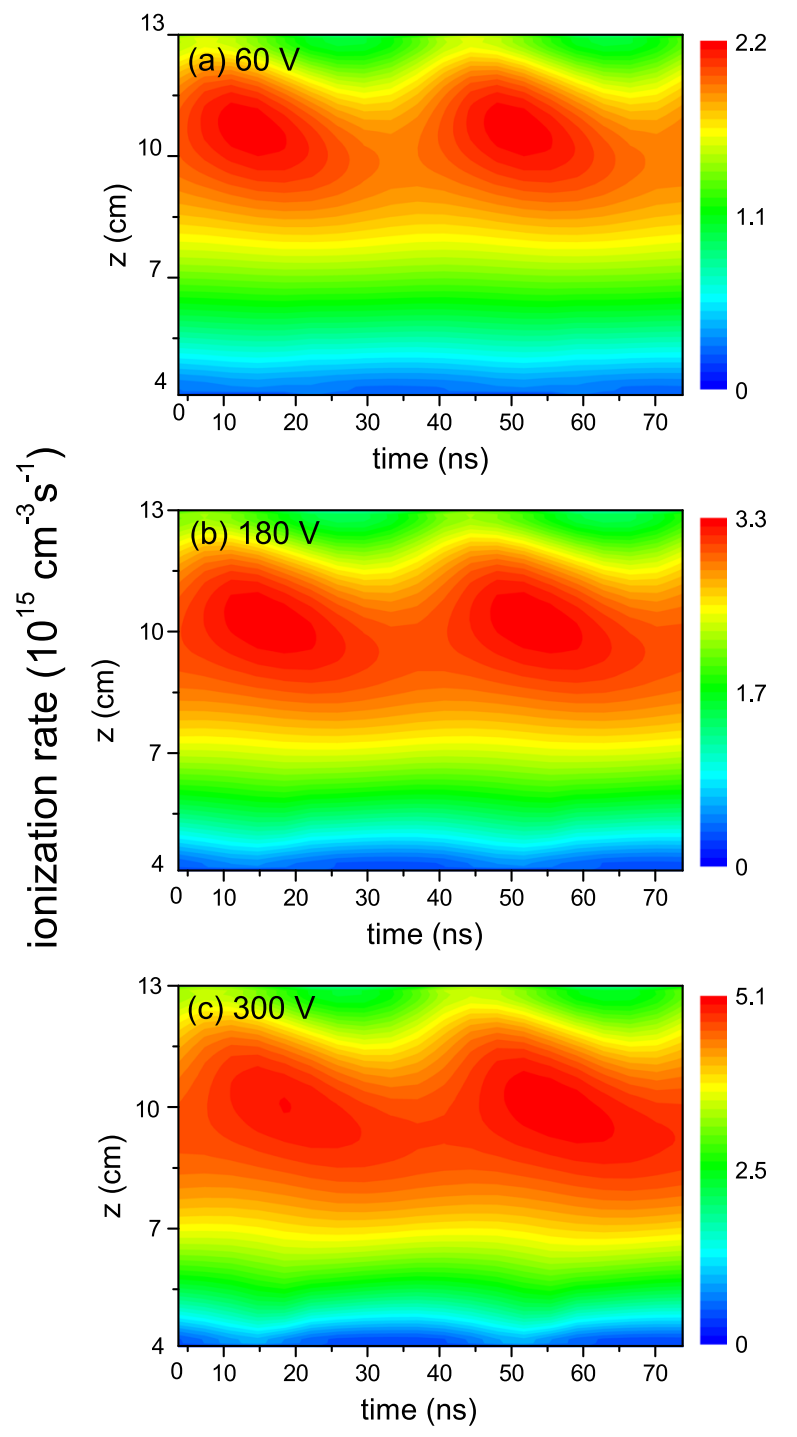

FIG. 14. (Color online) Spatiotemporal distributions of the ionization rate at $\mathrm{r}=7 \mathrm{~cm}$ at different bias voltages: (a) $60 \mathrm{~V}$, (b) $180 \mathrm{~V}$, (c) $300 \mathrm{~V}$, for an argon discharge sustained at an ICP current of $11 \mathrm{~A}$ and $13.56 \mathrm{MHz}$, with a bias frequency of $27.12 \mathrm{MHz}$.

respectively. In Fig. 13, the results are plotted in a timeframe of $500 \mathrm{~ns}$, corresponding to one cycle at $2 \mathrm{MHz}$. At this low bias frequency, the ionization primarily occurs under the dielectric window at the bias voltage of $60 \mathrm{~V}$ and is characterized by 14 peaks during one low frequency period [cf. Fig. 13(a)], due to the energetic electrons accelerated by the inductive field. As the bias voltage increases to $180 \mathrm{~V}$ [cf. Fig. 13(b)], the ionization is clearly modulated by the bias effect, which is strikingly different from the results obtained at $13.56 \mathrm{MHz}$. Indeed, the absolute values of the peaks oscillate as a function of time, and a minimum of the ionization rate appears near the bottom electrode, which indicates that the bias power starts to have a significant influence on the plasma generation. At the bias voltage of $300 \mathrm{~V}$, shown in Fig. 13(c), the modulation of the bias power becomes even more prominent. The peaks of the ionization rate caused by the inductive field heating are first remarkable at about $150 \mathrm{~s}$ (i.e., at maximum about $5.9 \times 10^{15} \mathrm{~cm}^{-3} \mathrm{~s}^{-1}$ ), and at a later time they become less prominent (i.e., around $4.4 \times 10^{15} \mathrm{~cm}^{-3} \mathrm{~s}^{-1}$ ). Besides, the maximum of the ionization rate appears near the bottom electrode, which means that the discharge is mainly sustained by the bias power under this condition.

The comparison of the ionization rate profiles at various bias voltages at the bias frequency of $27.12 \mathrm{MHz}$ is depicted in Fig. 14. During one low frequency period (74 ns for $13.56 \mathrm{MHz}$ ) at $60 \mathrm{~V}$, the ionization is again at maximum near the quartz window [Fig. 14(a)], which is similar to the case at $13.56 \mathrm{MHz}, 90 \mathrm{~V}$ [cf. Fig. 8(b)], except for the higher absolute values (i.e., in the order of $2.2 \times 10^{15} \mathrm{~cm}^{-3} \mathrm{~s}^{-1}$ ). At the bias voltage of 180 and $300 \mathrm{~V}$ [see Figs. 14(b) and 14(c)], the maxima become more intense with higher absolute values. Besides, the ionization rate exhibits two minima above the bias electrode. This is because the RF capacitive field induced by the bias power oscillates with time twice within one low frequency cycle. Furthermore, it is clear that the ionization rate distribution is different from that obtained at the bias frequency of $13.56 \mathrm{MHz}$ [Fig. 8(c)], which also demonstrates the different modulation of the bias effect at various frequencies.

\section{CONCLUSIONS}

In this paper, the MAPS-ICP solver combined with the MAPS-sheath module is employed to investigate the bias effect on the discharge mode transition and plasma characteristics in an argon inductively coupled plasma with a capacitive bias power. The different effects at various ICP currents, bias frequencies and bias voltages have been illustrated by examining the spatiotemporal averaged plasma density, the ratio of the absorbed bias power to the total power, as well as the distribution of the ionization rate.

From the simulation results, it is clear that the bias power influences the mode transition and plasma properties in a different way at various coil currents. Indeed, when the current is low, i.e., at $6 \mathrm{~A}$, the spatiotemporal averaged plasma density increases monotonically with bias voltage, due to the increasing bias power deposition. However, when the ICP current becomes higher (i.e., 11 and 13 A), a strikingly different trend is observed. The plasma density decreases first and then increases with bias voltage. This is because when the bias voltage increases slightly, the power dissipation caused by the ion acceleration becomes substantial and therefore gives rise to a decrease in the plasma density. However, as the bias voltage increases further, the power deposition induced by the bias source plays a dominant role in the plasma generation, and accordingly, it results in a higher plasma density.

The spatial distribution of the ionization rate also exhibits different profiles. When the bias source is switched off at the ICP current of $6 \mathrm{~A}$, the ionization rate has a broad maximum at the reactor center near the dielectric window. As the bias voltage increases, the peak moves toward the bottom electrode with higher absolute values, which indicates the discharge mode transition from inductive mode to capacitive mode. As the current increases to $11 \mathrm{~A}$ without a bias source, 
the maximum under the quartz window is more intense and more localized than that obtained at $6 \mathrm{~A}$, and the absolute value is higher. When the bias source is switched on, the maximum of the ionization rate again moves downwards, because of the pronounced capacitive bias power. At the high coil current of $13 \mathrm{~A}$, no significant effect of the bias voltage on the ionization rate distribution is detected, except for the absolute value, which indicates that the inductive power plays a dominant role under this discharge condition.

We also investigated the bias effect on the spatiotemporal distribution of the ionization rate at $\mathrm{r}=7 \mathrm{~cm}$. When no bias power is applied at $6 \mathrm{~A}$, a substantial ionization takes place in the bulk region with two peaks during one period. As the bias voltage increases, the ionization rate displays only one peak near the bottom electrode with higher values, which means the discharge is mainly sustained in the capacitive mode. However, when the ICP current increases to $11 \mathrm{~A}$ and especially $13 \mathrm{~A}$, the ionization rate is characterized by two peaks under the dielectric window at all selected bias voltages, which is typical for the inductive mode.

The different bias effects on the plasma characteristics have also been demonstrated at various bias frequencies. The evolution of the plasma density with bias voltage is similar at all bias frequencies investigated, and is characterized by first a drop and then a rise upon increasing bias voltage, but the minimum appears at different bias voltages, i.e., $120 \mathrm{~V}$ at $2 \mathrm{MHz}, 60 \mathrm{~V}$ at $13.56 \mathrm{MHz}$, and $90 \mathrm{~V}$ at $27.12 \mathrm{MHz}$. At the bias frequency of $2 \mathrm{MHz}$, the maxima of the ionization rate are closer to the dielectric window than those obtained at $27.12 \mathrm{MHz}$. Besides, the maxima of the ionization rate oscillate as a function of time at $2 \mathrm{MHz}$, which indicates the evident modulation of the bias effect.

\section{ACKNOWLEDGMENTS}

This work was supported by the Important National Science and Technology Specific Project (Grant No. 2011ZX02403-001), the National Natural Science Foundation of China (Grant Nos. 11175034, 11205025, and 11405019), the China Postdoctoral Science Foundation (Grant No. 2014M561219), the Fundamental Research Funds for Central Universities (Grant No. DUT14RC(3)071), the International Science and Technology Cooperation Program of China (Grant No. 2012DFG02150), the joint research project in the framework of the agreement between MOST and FWO, and the University of Antwerp BOF fellowship for Y.-R. Zhang.
${ }^{1}$ M. A. Lieberman and A. J. Lichtenberg, Principles of Plasma Discharges and Materials Processing, 2nd ed. (Wiley, New York, 2005).

${ }^{2}$ P. L. G. Ventzek, M. Grapperhaus, and M. J. Kushner, J. Vac. Sci. Technol., B 12, 3118 (1994).

${ }^{3}$ J. H. Keller, J. C. Forster, and M. S. Barnes, J. Vac. Sci. Technol., A 11, 2487 (1993).

${ }^{4}$ G. A. Hebner and P. A. Miller, J. Appl. Phys. 87, 7660 (2000).

${ }^{5}$ D. S. Wuu, C. R. Chung, Y. H. Liu, R. H. Horng, and S. H. Huang, J. Vac. Sci. Technol., B 20, 902 (2002).

${ }^{6}$ N. O. V. Plank, M. A. Blauw, E. W. J. M. van der Drift, and R. Cheung, J. Phys. D: Appl. Phys. 36, 482 (2003).

${ }^{7}$ S. Imai, J. Vac. Sci. Technol., B 26, 2008 (2008).

${ }^{8}$ S. Rauf, P. L. G. Ventzek, I. C. Abraham, G. A. Hebner, and J. R. Woodworth, J. Appl. Phys. 92, 6998 (2002).

${ }^{9}$ M. A. Sobolewski and J. H. Kim, J. Appl. Phys. 102, 113302 (2007).

${ }^{10}$ H. C. Lee, M. H. Lee, and C. W. Chung, Appl. Phys. Lett. 96, 071501 (2010).

${ }^{11}$ J. Schulze, E. Schungel, and U. Czarnetzki, Appl. Phys. Lett. 100, 024102 (2012).

${ }^{12}$ H. C. Lee, S. Oh, and C. W. Chung, Plasma Sources Sci. Technol. 21, 035003 (2012).

${ }^{13}$ H. C. Lee and C. W. Chung, Appl. Phys. Lett. 101, 244104 (2012).

${ }^{14}$ R. J. Hoekstra and M. J. Kushner, J. Appl. Phys. 79, 2275 (1996).

${ }^{15}$ H. Takekida and K. Nanbu, J. Phys. D: Appl. Phys. 38, 3461 (2005).

${ }^{16}$ S. Tinck, W. Boullart, and A. Bogaerts, J. Phys. D: Appl. Phys. 41, 065207 (2008).

${ }^{17}$ D. C. Kwon, W. S. Shang, M. Park, D. H. You, M. Y. Song, S. J. You, Y. H. Im, and J. S. Yoon, J. Appl. Phys. 109, 073311 (2011).

${ }^{18}$ D. Zhang and M. J. Kushner, J. Vac. Sci. Technol., A 19, 524 (2001).

${ }^{19}$ S. Rauf, J. Vac. Sci. Technol., B 22, 202 (2004).

${ }^{20}$ Y. R. Zhang, X. Xu, S. X. Zhao, A. Bogaerts, and Y. N. Wang, Phys. Plasmas 17, 113512 (2010).

${ }^{21}$ Y. R. Zhang, A. Bogaerts, and Y. N. Wang, J. Phys. D: Appl. Phys. 45, 485204 (2012).

${ }^{22}$ H. Y. Wang, W. Jiang, and Y. N. Wang, Plasma Sources Sci. Technol. 19, 045023 (2010).

${ }^{23}$ W. Jiang, H. Y. Wang, Z. H. Bi, and Y. N. Wang, Plasma Sources Sci. Technol. 20, 035013 (2011).

${ }^{24}$ S. X. Zhao, X. Xu, X. C. Li, and Y. N. Wang, J. Appl. Phys. 105, 083306 (2009).

${ }^{25}$ X. J. Si, S. X. Zhao, X. Xu, A. Bogaerts, and Y. N. Wang, Phys. Plasmas 18, 033504 (2011).

${ }^{26}$ J. D. Bukowski, D. B. Graves, and P. Vitello, J. Appl. Phys. 80, 2614 (1996).

${ }^{27}$ P. L. G. Ventzek, R. J. Hoekstra, and M. J. Kushner, J. Vac. Sci. Technol., B 12, 461 (1994).

${ }^{28}$ M. A. Liberman, IEEE Trans. Plasma Sci. 16, 638 (1988).

${ }^{29}$ V. A. Godyak and N. Sternberg, Phys. Rev. A 42, 2299 (1990).

${ }^{30}$ M. J. Grapperhaus and M. J. Kushner, J. Appl. Phys. 81, 569 (1997).

${ }^{31}$ P. A. Miller and M. E. Riley, J. Appl. Phys. 82, 3689 (1997).

${ }^{32}$ T. Panagopoulos and D. J. Economou, J. Appl. Phys. 85, 3435 (1999).

${ }^{33}$ E. A. Edekberg and E. S. Aydil, J. Appl. Phys. 86, 4799 (1999).

${ }^{34}$ D. Bose, T. R. Govindan, and M. Meyyappan, J. Appl. Phys. 87, 7176 (2000).

${ }^{35}$ Z. L. Dai, Y. N. Wang, and T. C. Ma, Phys. Rev. E 65, 036403 (2002).

${ }^{36}$ D. Rapp and P. Englander-Golden, J. Chem. Phys. 43, 1464 (1965).

${ }^{37}$ W. L. Morgan, "Kinema Research \& Software," http://www.lxcat.laplace.univ-tlse.fr/.

${ }^{38}$ D. Ton-That and M. R. Flannery, Phys. Rev. A 15, 517 (1977).

${ }^{39}$ F. Gao, S. X. Zhao, X. S. Li, and Y. N. Wang, Phys. Plasmas 16, 113502 (2009).

${ }^{40}$ D. P. Lymberopoulos and D. J. Economou, J. Appl. Phys. 73, 3668 (1993). 